\title{
Anticancer Effect of Natural Product Sulforaphane by Targeting MAPK Signal through miRNA-1247-3p in Human Cervical Cancer Cells
}

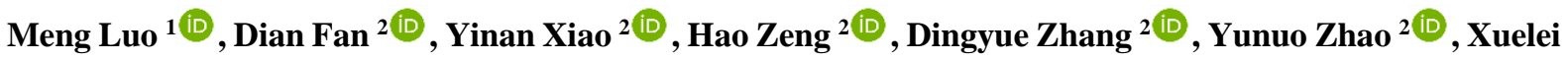 \\ Ma 1 ,*iD \\ 1 Department of Biotherapy, State Key Laboratory of Biotherapy and Cancer Center, West China Hospital, Sichuan \\ University, Chengdu, Sichuan 610041, P.R. China; luomeng_1017@163.com (L.M.); \\ 2 West China Medical School, West China Hospital, Sichuan University, Chengdu, Sichuan 610041, P.R. China; \\ fan-dian@foxmail.com (F.D.); yinan_xiao@163.com $\quad$ (X.Y.N.); $\quad 13540285357 @ 163 . c o m \quad$ (Z.H.); \\ 2017141082017@stu.scu.edu.cn (Z.D.Y.); 2087247019@qq.com (Z.Y.N.); \\ * Correspondence: drmaxuelei@gmail.com;
}

Received: 11.06.2020; Revised: 3.07.2020; Accepted: 5.07.2020; Published: 9.07.2020

\begin{abstract}
The prognosis of cervical cancer remains poor. Sulforaphane, an active ingredient from cruciferous plants, has been identified as a potential anticancer agent in various cancers. However, there is little information about its effect on cervical cancer. Here, we conducted a present study to uncover the effect and the potential mechanisms of sulforaphane on cervical cancer. HeLa cells were treated with sulforaphane, and cell proliferation and apoptosis were assessed by Cell Counting Kit-8, Western blot, flow cytometry, and immunofluorescence. Then, next-generation sequencing (NGS) and bioinformatics tools were used to analyze mRNA-seq, miRNA-seq, and potential pathways. Finally, qRT-PCR, Cell Counting Kit-8, flow cytometry, small RNAs analysis, and Western blot were performed to evaluate the biological function of miR1247-3p and MAPK pathway in HeLa cell lines. Sulforaphane significantly suppressed the viability and induced apoptosis of HeLa cells. NGS and bioinformatics analysis showed sulforaphane exerted its anti-tumor activities through miR1247-3p and the MAPK signaling pathway. Further analysis suggested that sulforaphane could activate MAPK pathway via down-regulating the expression of miR-1247-3p. Sulforaphane inhibited proliferation and promoted apoptosis of HeLa cells via down-regulation of miR-1247-3p and activating the MAPK pathway. These findings provide preliminary experimental evidence for the treatment of cervical cancer with sulforaphane.
\end{abstract}

Keywords: Sulforaphane; Cervical cancer; Next-generation sequencing; Kyoto Encyclopedia of Genes and Genomes; MiR-1247-3p; MAPK pathway.

List of Abbreviations: CCK8 = cell counting kit 8, DMSO = dimethyl sulfoxide, GO = Gene Ontology, KEGG = Kyoto Encyclopedia of Genes and genomes, MAPK = mitogen-activated protein kinase, miRNA $=$ microRNA, NGS $=$ next-generation sequencing, qRT-PCR $=$ quantitative real-time polymerase chain reaction, $\mathrm{PI}=$ propidium iodide

(C) 2020 by the authors. This article is an open-access article distributed under the terms and conditions of the Creative Commons Attribution (CC BY) license (https://creativecommons.org/licenses/by/4.0/).

\section{Introduction}

Cervical cancer has become the fourth most common malignancy among women worldwide due to its high incidence and mortality rate[1] [2]. There are around 528,000 newlydiagnosed cervical cancer cases and 266,000 deaths every year, according to the latest global cancer statistics [3]. To date, effective treatments for patients with cervical cancer include surgery, chemotherapy, and radiotherapy[4]. Given many potential adverse side effects in these 
traditional treatments, so it is worthwhile to search for a novel effective treatment for cervical cancer [5].

Sulforaphane, an herbal isothiocyanate typically abundant in cruciferous vegetables such as broccoli, cauliflower, and cabbage, has many biological effects such as antiinflammation, anti-oxidation [6]. In particular, due to its anticarcinogenic role in pancreatic cancer, it has been noteworthy recently [7]. The antiproliferative and radiosensitizing properties for head and neck tumors also have been reported [8]. Although previous studies have identified the anti-tumor potential of sulforaphane in various kinds of cancers $[9,10]$, the role of sulforaphane in cervical cancer has not been well recognized. MicroRNAs (miRNAs) are short, non-coding RNAs that play important roles in regulating many biological processes involving almost all aspects of cell physiology [11,12]. Typically, they regulate protein expression levels in physiological and pathophysiological processes by conjugating with complementary sequences of their target mRNAs[13,14]. As there are around 1000 miRNAs in the human genome, which modulate approximately one-third of the human genes[15], miRNAs are the most abundant regulators[16].

Accumulating evidence has shown that sulforaphane exerts its therapeutic effects through modulating the expression of cancer-related miRNAs on a variety of cancers, including colon cancer[17], lung cancer[18], gastric cancer[19], pancreatic cancer[20], skin cancer[21], acute myeloid leukemia[22], and nasopharyngeal cancer[23]. Besides, the previous study has demonstrated that miR-3156-3p, negatively associated with the incidence of HPV-positive cervical cancer, acted as a tumor-suppressive miRNA[24]. Furthermore, targeting MACC1, miR-338-3p could regulate the proliferation of cervical cancer cells via the MAPK pathway[25]. However, no elaborate research has found the association between miRNAs and sulforaphane in inhibiting cervical cancer until now. Therefore, given the anti-tumor capability of sulforaphane, we aimed at validating the anticancer activities and mechanisms of sulforaphane in cervical cancer, including downstream target miRNA and relevant signal pathway initiated by the miRNA.

In our study, we found that miR-1247-3p was highly related to both the apoptosis rate of cervical cancer cells cultured with sulforaphane and the activation of the MAPK pathway. Based on the high throughput sequencing and bioinformatics analysis, we ultimately disclosed the role of the miR-1247-3p-induced MAPK signal pathway in inhibition of cervical cancers treated with sulforaphane.

\section{Materials and Methods}

\subsection{Materials.}

Sulforaphane was purchased from MedChem Express (\#HY-13755, MCE, Monmouth Junction, NJ, USA) and dissolved in DMSO (Sigma, Saint Louis, USA) at the concentration of $10 \mathrm{mM}$ and protected from light. It stored as small aliquots at $-20^{\circ} \mathrm{C}$ for long term preservation. The primers for miR-12315 (HmiRQP4717), miR-1247-3p(HmiRQP3407), miR33b-3p(HmiRQP0431), miR-320-5p (HmiRQP4731) and small nuclear RNA-U6 (RNU6, \#HmiRQP9001) were obtained by Genecopoeia (Rockville, MD, USA). The sequence of miR12315 inhibitor was: AUGGUGUCGGAAAAUCG UAGCCGAAGACACCUCGGACGA GAGACCGA CACCGCCA. The sequence of miR-1247-3p mimic was: CCCCGGGAACGUCGAGACUGGAGC. The sequence of miR-320a-5p inhibitor was: CGGAAGAGAAGGGCCAAGAAGG. The sequence of miR-33b-3p mimic was: 
CAGUGCCU CGGCAGUGCAGCCC. The sequence of inhibitor NC was: CAGUACUUUUGUGUAGUACAA. The sequence of mimic NC was: UUGUACUACACAAAAGUACUG.

\subsection{Cell culture.}

Human cervical cancer cell lines HeLa was purchased from the American Model Culture Collection (ATCC), which was preserved by the State Key Laboratory of Biotherapy of Sichuan University. The cell was cultured in Dulbecco's modified Eagle medium (DMEM) supplemented with 10\% fetal bovine serum (FBS; Gibco), 100 unit $/ \mathrm{mL}$ penicillin( Beyotime, $\mathrm{SH}$, China), and $100 \mathrm{mg} / \mathrm{mL}$ streptomycin (Beyotime). These cells were cultured in a humid chamber at $37^{\circ} \mathrm{C}$ under $5 \% \mathrm{CO} 2$ in the atmosphere.

\subsection{Cell viability assay.}

CCK8 kit was used to detect cell proliferation. Briefly, $5 \times 10^{3}$ cells were plated in96 well plates. After attachment overnight, cells were treated with sulforaphane $(0,10,20,40 \mu \mathrm{M})$ for $24 \mathrm{~h}$, and then incubated in $10 \% \mathrm{CCK}-8$ for another $2 \mathrm{~h}$. The cells in the $0 \mu \mathrm{M}$-treated group were cultured with the same volume of DMSO as the $40 \mu \mathrm{M}$-treated group. The cell proliferation rate was measured at $450 \mathrm{~nm}$ absorbance. The experiment was carried out three times.

\subsection{Transcriptome sequencing and analysis.}

HeLa cells were harvested for RNA extraction after $8 \mathrm{~h}$ of sulforaphane treatment at a concentration of $40 \mu \mathrm{M}$. The cells incubated with the same volume of DMSO as the sulforaphane $(40 \mu \mathrm{M})$-treated group were set as the control. Each group included three samples. Trizol Reagent (Invitrogen, San Diego, CA, USA) was used to isolate total RNA. Illumina HiSeq 2500 platform was utilized to perform transcriptome sequencing, and 150bp paired-end raw reads were generated. Illumina sequencing was carried out at Novogene, Beijing, China. The expression level of each transcript was measured as the number of clean reads mapped to its reference sequence (GRCh 38). Kallisto[26] was adopted to align and quantify clean reads, genes expressed in at least 1 sample were defined as detected genes. Differential expression analysis was performed through the DEseq2(v 1.24.0) R package [27], DEGs were selected out based on the interval of $p<0.05 \& \mid \log 2$ (fold change) $\mid>1$. Enrichment of pathways was performed by KOBAS 3.0[28] with the corresponding groups of DEGs. The hypergeometric test method was Fisher's exact test, and the FDR correction method was Benjamini and Hochberg.

\subsection{Small RNA Sequencing and Analysis.}

Small RNAs were sequenced by Illumina Hiseq 2500/2000 platform, and 50 bp singleend reads were generated. Clean data were obtained by removing adaptor sequences, collapsing reads with the same sequence, and reads with less than 15 bases in length from raw data. Then, clean data were analyzed based on the miRDeep2 (2.0.0.8)[29]. Small RNA reads were mapped to miRNA precursors from miRBASE release 22.1[30] by miRDeep2, which allowed zero mismatches. Mapped reads were quantified by miRDeep2. Reads were matched to human miRNA precursors using bowtie2[31], and 1520 miRNAs were detected. Analysis of the differential expressed miRNAs(DEMs) between sulforaphane-untreated and sulforaphane- 
treated groups was performed by the DEseq2 R package. $\mathrm{P}<0.05 \& \mid \log 2$ (fold change) $\mid>2$ was set to as the significance threshold in this test. The target genes of DEMs were predicted by TargetScanHuman(v 7.2)[32].The Enrichment of KEGG pathways was performed by KOBAS 3.0 with the target genes.

\subsection{Flowcytometry.}

FITC-Annexin V/PI Detection Kit I (BD Biosciences, San Jose, CA) was used for quantifying the apoptotic cell rate after sulforaphane treatment. The HeLa cells $\left(5 \times 10^{5}\right.$ cells/well) were plated in 6-well plates. After $24 \mathrm{~h}$ incubation, the cells were cultured with different concentrations of sulforaphane for $24 \mathrm{~h}$. Then the cells were collected and incubated with Annexin V-FITC for $5 \mathrm{~min}$ in the dark and then stained with PI for $5 \mathrm{~min}$. The sample was sorted by NovoCyte flow cytometry. Data were analyzed by NovoExpress 1.1.2 software.

\section{7. miRNA transfection.}

MiR-1247-3p mimic and its scrambled control (NC) were both purchased from GenePharma (Shanghai, China). For transfection, the cells were seeded at a density of $4 \times 10^{4}$ cells per well into 6-well culture plates. As the cells reached 50-80\% confluence, transient transfection was performed by using Lipofectamine ${ }^{\mathrm{TM}} 3000$ (Invitrogen) according to the manufactures' protocol. The transfection was stopped after 24 hours, and cells were collected for subsequent experiments, including cell viability and apoptosis assays. In cell viability assay, the cells transfected with different miRNA mimics were administrated with sulforaphane $(0$, 10,20 , and $40 \mu \mathrm{M}$ ) for $24 \mathrm{~h}$. In apoptosis assay, the flow cytometry detected the apoptotic cell rate of sulforaphane-treated cells $(0,40 \mu \mathrm{M})$ for $24 \mathrm{~h}$. The cells in the sulforaphane $(0 \mu \mathrm{M})$ treated group(control group) were incubated with the same volume of DMSO as the sulforaphane $(40 \mu \mathrm{M})$-treated group.

\subsection{Immunofluorescence.}

HeLa cells were seed onto a 14-mm cover glass in 24-well plate and incubated overnight. Cells were treated by different concentrations of sulforaphane for $48 \mathrm{~h}$. Then the culture medium was removed, and cells were washed twice with PBS, fixed with 4\% paraformaldehyde for $10 \mathrm{~min}$ and washed three times. The unspecific binding sites were blocked with PBST containing 1\% BSA and $0.05 \%$ Triton X-100. Subsequently, cells were incubated with primary antibody overnight at $4{ }^{\circ} \mathrm{C}$. The secondary antibodies combined with FITC were used. DAPI was performed to stained cell nuclei, and the samples were examined with fluorescence microscopy.

\section{9. $R T-P C R$.}

To confirm the bioinformatics analysis, total RNA was extracted by using RNA Extraction Kit (TIANGEN BIOTECH, DP419) after treatment with or without sulforaphane $(40 \mu \mathrm{M})$ for $8 \mathrm{~h}$, and reverse transcribed to cDNA by using Prime Script RT Kit (Takara, RR036) according to the manufacturers' instructions. The qPCR array was performed by using SYBR Select Master Mix (Invitrogen, 4472908) with specific gene primers in StepOnePlus PCR System (Thermo). RT-qPCR was conducted by the following conditions: $95^{\circ} \mathrm{C}$ for 15 min, followed by 40 cycles of $94^{\circ} \mathrm{C}$ for $20 \mathrm{~s}$ and $60^{\circ} \mathrm{C}$ for $34 \mathrm{~s}$. RNU6 was used as an internal reference. The target genes expression levels were measured by using the $2^{-{ }^{\mathrm{Ct}}}$ method. 


\subsection{Western blot.}

The harvested cells were washed twice with a pre-cooled PBS buffer. Cellular proteins were extracted in RIPA lysis buffer (Biosharp, Guangzhou, GD, China), including protease inhibitor cocktail (Millipore) over ice for 30 minutes. The supernatant was gathered by centrifuging at $13000 \mathrm{rpm}$ for $15 \mathrm{~min}$ at $4^{\circ} \mathrm{C}$. The protein concentrations of samples were evaluated by BCA protein assay kit (Beyotime, China). Afterwards, the protein extracts were separated in SDS-PAGE gel and transferred onto nitrocellulose (NC) membranes (Merck Millipore, MS, USA). Then, the membranes were blocked at room temperature for $1.5 \mathrm{~h}$ and incubated with proper primary antibody and the corresponding secondary antibody. These primary antibodies were purchased from HuaBio (Hangzhou, ZJ, China), including antiCytochrome C antibody (\#ET1610-16), anti-Bax antibody (\#ET1603-34), anti- $\beta$-Actin antibody (\#R1207-1). The other primary antibodies were purchased from Cell Signaling Technology, including anti-p-ERK (\#4370), anti-ERK(\#4695), (anti-p-JNK(\#9255), anti JNK(\#9252), anti-p-P38(\#4511) and anti -P38(\#8690). The specific protein bands were detected via chemiluminescence detection.

\subsection{Statistical analysis.}

All the statistics were carried out in GraphPad Prism 7 and 8. All data are shown as mean \pm SD. A two-tailed Student's t-test was used to analyze statistical significance. The statistic differences are shown as $* \mathrm{p}<0.05, * * \mathrm{p} b<0.01, * * * \mathrm{p}<0.001$, ns, no significance.

\section{Results and Discussion}

\subsection{Sulforaphane inhibited the proliferation and induced apoptosis of HeLa cells.}

To evaluate the effect of sulforaphane on cell viability, the HeLa cell was cultured with sulforaphane of different concentrations $(0,10,20,40$, and $60 \mu \mathrm{M})$ for $24 \mathrm{~h}$. According to the results of our CCK-8 array, sulforaphane suppressed the viability of HeLa cells in a concentration-dependent manner (Fig. 1A).
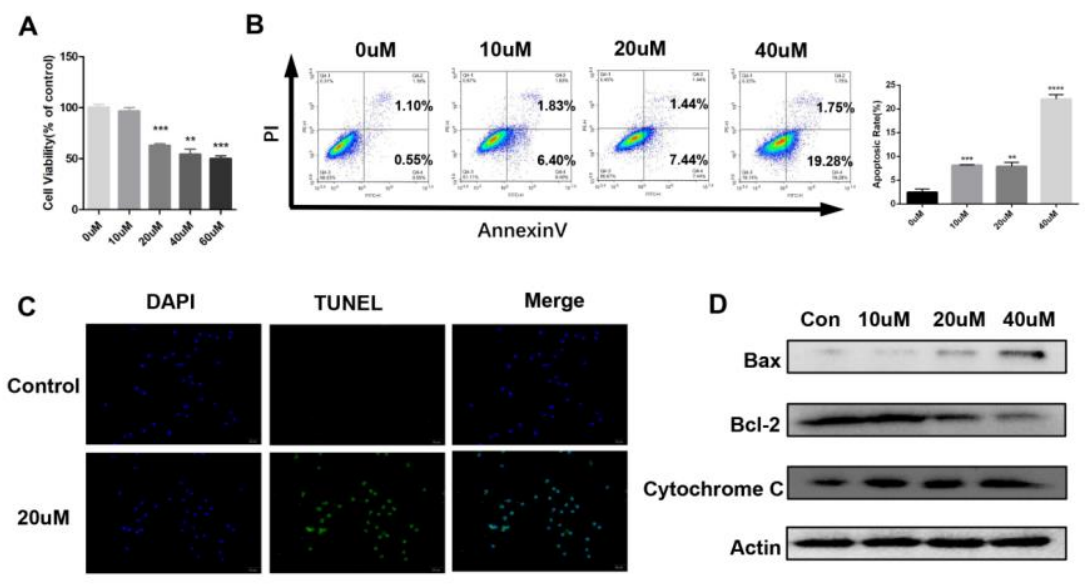

Figure 1. Sulforaphane inhibited the proliferation and induced apoptosis of HeLa cells. (A) The viability of cells pre-treated with sulforaphane $(0,10,20,40,60 \mu \mathrm{M})$ was detected. (B) Flow cytometric analysis of HeLa cells apoptosis using the Annexin V/PI dual-labeling technique after sulforaphane treatment $(0,10,20,40 \mu \mathrm{M}))$ for $24 \mathrm{~h}$. (C) Apoptosis of the sulforaphane-treated group and control group were calculated by TUNEL assays. (D) Protein level expression of apoptosis in cell pre-treated with $0,10,20$, and $40 \mu \mathrm{M}$ sulforaphane. Data are shown as mean \pm SEM $(\mathrm{n}=3) ; * \mathrm{P}<0.05, * * \mathrm{P}<0.01$, *** $\mathrm{P}<0.001$ vs. control group. 
As shown in Fig. 1B, the flow cytometry analysis showed the apoptotic rate of HeLa cells of $40 \mu \mathrm{M}$ treatment after $24 \mathrm{~h}$ remarkably increased compared with the rate of $0 \mu \mathrm{M}$ after 24h. Moreover, the apoptosis rate determined by the TUNEL assay increased (Fig. 1C). To further investigate apoptosis induced by sulforaphane, we analyzed the expression of Bcl-2, Bax, and Cytochrome C. As demonstrated in Fig. 1D, the expression level of anti-apoptotic Bcl-2 decreased. At the same time, pro-apoptotic Bax and Cytochrome $\mathrm{C}$ were up-regulated in a dose-dependent manner, which contributed to an increase in the Bax/Bcl-2 expression ratio. All of these proteins were crucial molecular regulators in the mitochondrial-mediated apoptotic pathway [33]. These data indicated that sulforaphane could inhibit the proliferation and induce the apoptosis of HeLa cells.

\subsection{An integrated analysis of altered mRNAs and miRNAs via high-throughput sequencing.}

According to the previous results, transcriptome sequencing and bioinformatics analysis were performed to the HeLa cells treated with sulforaphane at a concentration of 40 $\mu \mathrm{M}$ for $8 \mathrm{~h}$. Next-generation sequencing was applied to identify a large number of altered mRNAs and miRNAs after treatment. The analysis of transcription sequencing data described that a total of 21,582 expressed genes were detected.
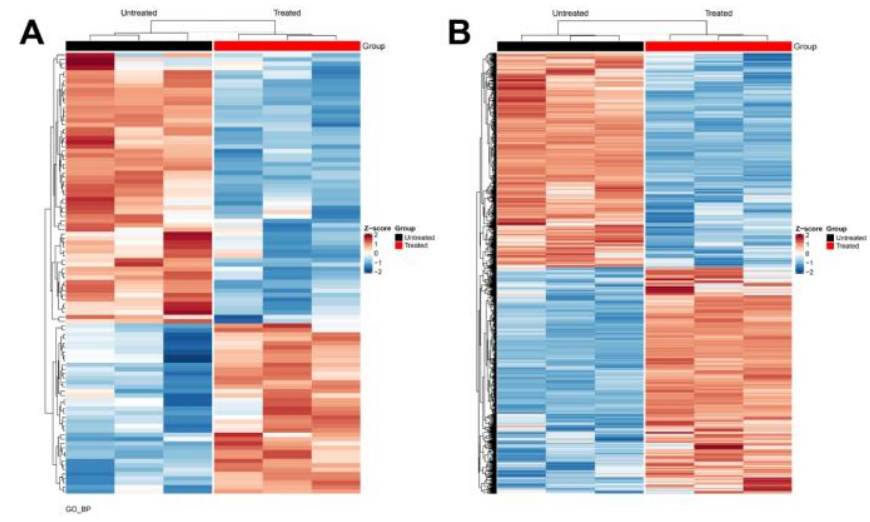

C
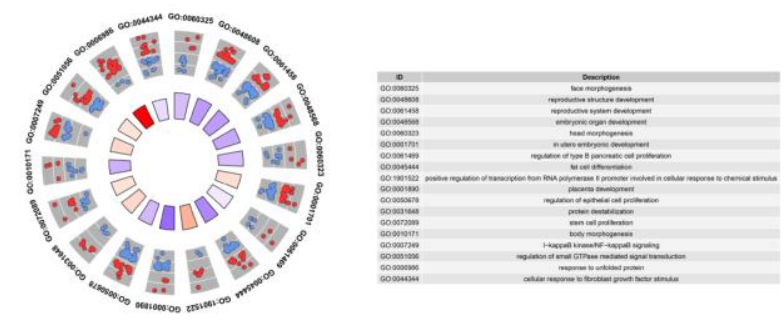

Et. - - - - -

Figure 2. High throughputs RNA sequencing of the sulforaphane treatment group and control group. (A) Heatmap of the count data of miRNAs that expression changed significantly $(\mathrm{P}<0.05)$ in HeLa cells treated with sulforaphane 40uM for 8 hours $(n=3)$. (B) Hierarchical clustering heatmap of TPM data of significantly changed genes after sulforaphane treatment. The color density indicates the average expression of a given gene, with each row normalized by z-score. $(\mathbf{C}) \mathrm{GO}$ plot of genes with different expression levels $(\mathrm{P}<0.05)$ of the sulforaphanetreated group in the biological process category of GO enrichment analysis. Red and blue dots in the scatter plot of outer wheel show up-regulated genes and downregulated genes, respectively; the inner wheel demonstrates the z-score.

The expression level of 7838 genes changed significantly $(\mathrm{P}<0.05)$ in the sulforaphane-treated group compared with the control group (Fig 2A). Ultimately, 2041 DEGs were identified by the DEseq2(v 1.24.0) R package. After the analysis of miRNA sequencing data, a total of 1588 miRNAs were spotted. Then the differential expression analysis of these detected miRNAs was performed between the sulforaphane-treated group and the control group 
(Fig 2B). Finally, 101 significantly changed miRNAs were found $(\mathrm{P}<0.05)$, which contained 39 up-regulated miRNAs and 62 downregulated miRNAs. To better determine the function of DEGs after sulforaphane expose, gene ontology (GO) enrichment analysis was used to find significantly enriched terms, leading to a more comprehensive understanding of the effects sulforaphane exerting on HeLa cells (Fig 2C). The results displayed that many biological processes were significantly altered after sulforaphane treatment, including response to unfolding protein (GO: 0006986), reproductive structure development (GO: 0048608), reproductive system development (G0:0061458), embryonic organ development(GO: 0048568).

3.3. Upregulation of miR-1247-3p promoted cell proliferation and induced anti-apoptosis in HeLa cells.

Several studies suggested that sulforaphane initiated its anti-tumor and antiinflammatory activities by regulating several miRNAs[23,34-37]. To further explore the role of sulforaphane, we evaluated miRNAs expression via high throughput RNA sequencing. Further, we identified the differential expression level of the miRNAs by the volcano plot in both the control group and the experiment group, as shown in Fig. 3A. Among the four significantly differentially expressed miRNAs, two miRNAs were up-regulated (miR-320a-5p, miR-12135), and the other two miRNAs were downregulated (miR-1247-3p, miR-33b-3p). qPCR was used to confirm the differential miRNA expression.
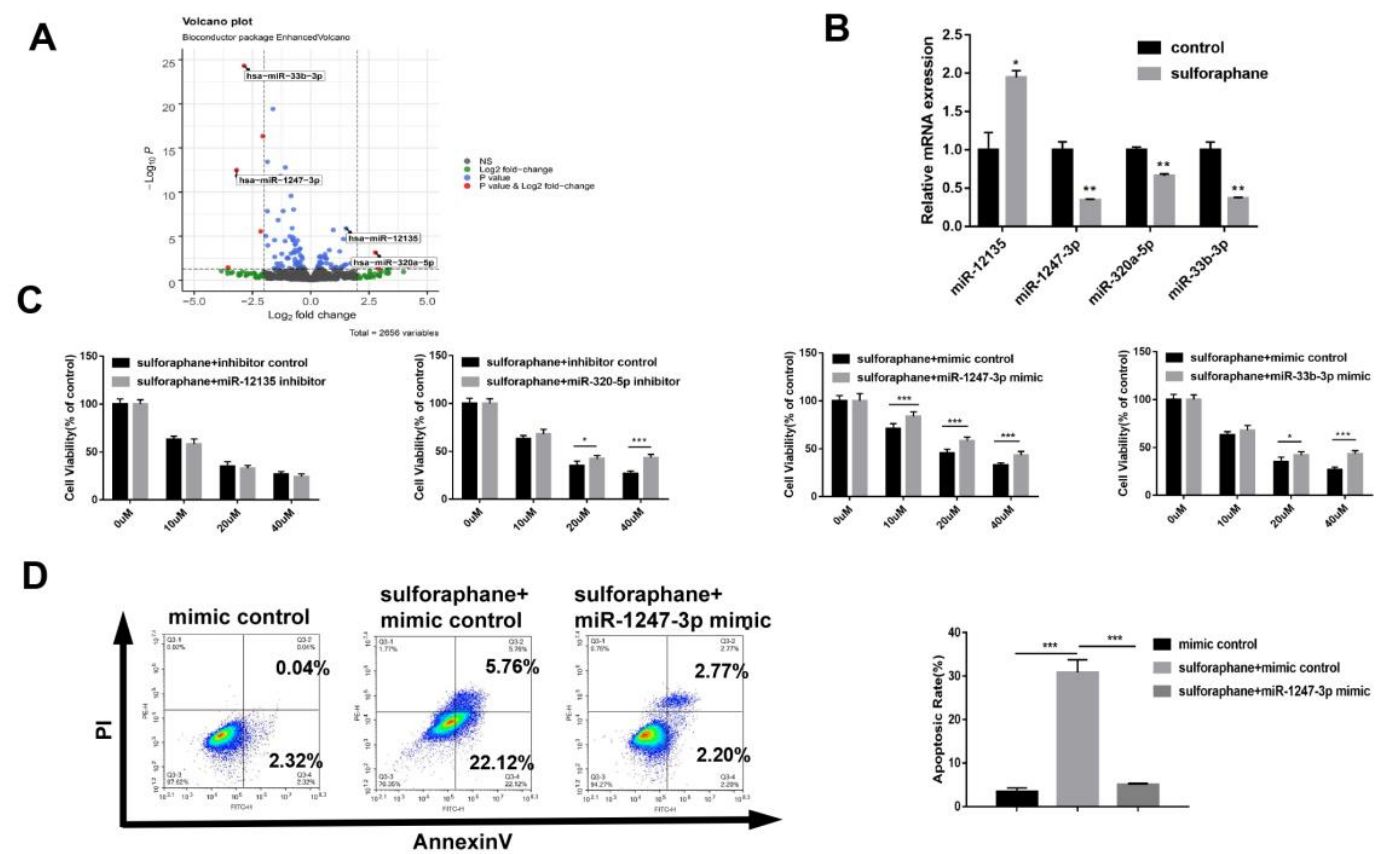

Figure 3. MiR-1247-3p was validated to regulate sulforaphane-induced apoptosis in HeLa cells. (A) A Volcano plot of miRNA microarray analysis, which was described with the $\log 2$ (fold change) of miRNAs expression level and $\mathrm{p}$-value based on $-\log 10$. The red dots mean the DEMs based on $\mathrm{p}<0.05$ (represented by black horizontal line) and 4-fold expression difference (represented by two black vertical lines), and the top changed four DEMs were labeled. (B) Relative miRNA expression in HeLa cells after sulforaphane treatment $(40 \mu \mathrm{M})$. (C) The viability of HeLa cells transfected with top changed four DEMs. (D) Flow cytometry analysis of apoptosis rate between the control group and miR-1247-3p transfected group by using the Annexin V/PI dual-labeling technique after 40uM sulforaphane treatment for $24 \mathrm{~h}$. Data are shown as mean $\pm \mathrm{SEM}(\mathrm{n}=3) ; * \mathrm{P}<0.05$, ** $\mathrm{P}<0.01$, *** $\mathrm{P}<0.005$. 
The expression of these miRNAs, except for miR-320a-5p, was consistent with RNAsequencing results (Fig.3B). Next, we tried to examine whether the four miRNAs would impact tumor cell proliferation. The result of the CCK-8 array exhibited that miR-1247-3p mimic could significantly increase HeLa cell proliferation (Fig.3C). Additionally, flow cytometry results confirmed that miR-1247-3p mimic dramatically inhibited cell apoptosis induced by sulforaphane(Fig. 3D). Thus, these results suggested miR-1247-3p could contribute to the antiapoptotic effect of sulforaphane on HeLa cells.

\subsection{Sulforaphane activated MAPK signaling pathway through downregulating miR-1247-3p.}

To find the mechanism of sulforaphane-induced apoptosis and further investigate the functions of miR-1247-3p, 2725 target genes were obtained (SupplyTable1) to predict the potential target pathways of miR-1247-3p (Fig 4A). Then KEGG pathway analysis was also carried out to explore the signaling pathways of the DEGs between sulforaphane-untreated and treated groups. The top 10 significantly enriched pathways are shown in Fig 4B, and the results are shown in SupplyTable 2. Some studies have revealed that sulforaphane exerted anti-tumor and anti-inflammatory effects via regulating MAPK signaling pathway[38-43].
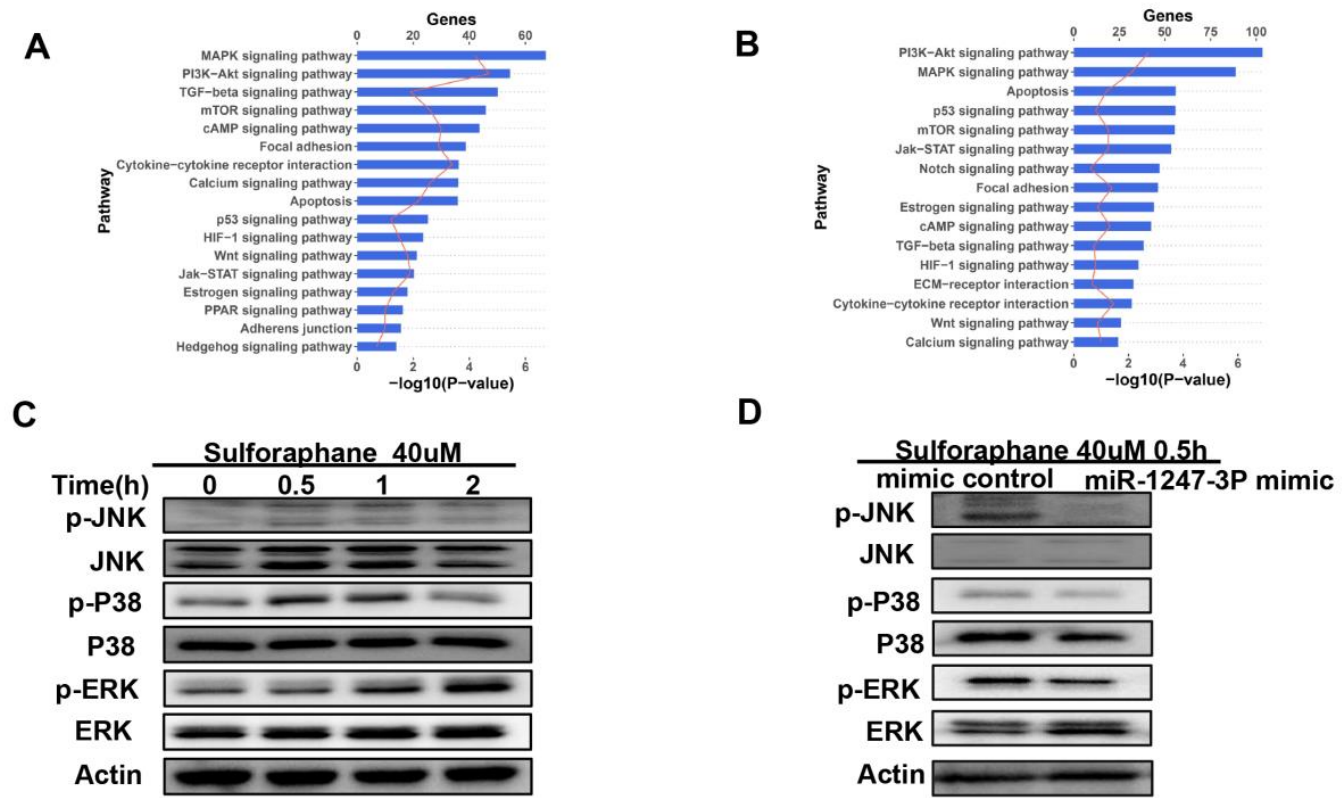

Figure 4 Sulforaphane activated MAPK signaling pathway via downregulating miR-1247-3p. (A) KEGG pathway enrichment analysis for target genes of miR-1247-3p, the top 10 significantly enriched pathways are presented. (B) Kyoto Encyclopedia of Genes and Genomes (KEGG) pathway enrichment analysis for DEGs between the sulforaphane treatment group and control group, the top 10 significantly enriched pathways are selected. For each KEGG pathway, the blue bar shows the p-value based on $-\log 10$ of the pathway, and the red line present hit genes number. (C) Western blot analysis of protein level of MAPK pathway in HeLa cells treated with $40 \mathrm{uM}$ of sulforaphane for $0.5 \mathrm{~h}, 1 \mathrm{~h}$, and $2 \mathrm{~h}$. Expressions of all the proteins were up-regulated with increasing time. (D) Protein expression levels of main factors in MAPK pathway after treatment with sulforaphane with or without miR-1247-3p mimic transfection.

So we tried to examine whether miR-1247-3p was implicated in the regulatory effect of sulforaphane via MAPK pathway on HeLa cells. The results showed that sulforaphane remarkably elevated the phosphorylation levels of JNK, P38, ERK (Fig. 4C). To further confirm these results, we detected JNK, P38, ERK protein levels between the mimic control group and miR-1247-3p mimic group. Results in Fig. 4D showed that the expression of p-JNK, 
p-P38, and p-ERK in cells transfected with miR-1247-3P mimic was much lower than that in mimic control-transfected cells. Therefore, these results implied that sulforaphane might downregulate miR-1247-3p to activate MAPK signaling pathway.

\section{Conclusions}

In summary, high throughput sequencing was applied in sulforaphane-treated HeLa cells, and we identified miR1247-3p downregulation was implicated in the pro-apoptosis effect of sulforaphane. Additionally, miR1247-3p-driven MAPK signaling pathway might be attributable to the apoptosis of HeLa cells. Based on the aforementioned mechanism, sulforaphane could serve as a promising anticancer agent in the treatment of cervical cancer.

\section{Funding}

This research received no external funding.

\section{Acknowledgments}

Yunuo Zhao and Dian Fan performed the molecular biology experiments. Meng Luo, Yinan Xiao, and Dingyue Zhang wrote the draft. Hao Zeng performed the miRNA chip analysis. Xuelei Ma designed the research and provided fund support.

\section{Conflicts of Interest}

The authors declare no conflict of interest.

\section{Data Availability Statement}

The raw data of next-generation sequencing used to support the findings of this study are available from the corresponding author upon request.

\section{References}

1. Arbyn, M.; Weiderpass, E.; Bruni, L.; de Sanjose, S.; Saraiya, M.; Ferlay, J.; Bray, F. Estimates of incidence and mortality of cervical cancer in 2018: a worldwide analysis. Lancet Glob Health 2020, 8, e191-e203, https://doi.org/10.1016/S2214-109X(19)30482-6.

2. Cohen, P.A.; Jhingran, A.; Oaknin, A.; Denny, L. Cervical cancer. Lancet 2019, 393, 169-182, https://doi.org/10.1016/S0140-6736(18)32470-X.

3. Ferlay, J.; Soerjomataram, I.; Dikshit, R.; Eser, S.; Mathers, C.; Rebelo, M.; Parkin, D.M.; Forman, D.; Bray, F. Cancer incidence and mortality worldwide: sources, methods and major patterns in GLOBOCAN 2012. Int J Cancer 2015, 136, E359-386, https://doi.org/10.1002/ijc.29210.

4. Sawaya, G.F.; Smith-McCune, K.; Kuppermann, M. Cervical Cancer Screening: More Choices in 2019. JAMA 2019, 321, 2018-2019, https://doi.org/10.1001/jama.2019.4595.

5. Rachmadi, L.; Siregar, N.C.; Kanoko, M.; Andrijono, A.; Bardosono, S.; Suryandari, D.A.; Sekarutami, S.M.; Hernowo, B.S. Role of Cancer Stem Cell, Apoptotic Factor, DNA Repair, and Telomerase Toward Radiation Therapy Response in Stage IIIB Cervical Cancer. Oman Med J 2019, 34, 224-230, https://doi.org/10.5001/omj.2019.43.

6. Singh, K.; Connors, S.L.; Macklin, E.A.; Smith, K.D.; Fahey, J.W.; Talalay, P.; Zimmerman, A.W. Sulforaphane treatment of autism spectrum disorder (ASD). Proc Natl Acad Sci U S A 2014, 111, 1555015555, https://doi.org/10.1073/pnas.1416940111.

7. Rodova, M.; Fu, J.; Watkins, D.N.; Srivastava, R.K.; Shankar, S. Sonic hedgehog signaling inhibition provides opportunities for targeted therapy by sulforaphane in regulating pancreatic cancer stem cell selfrenewal. PLoS One 2012, 7, https://doi.org/10.1371/journal.pone.0046083.

8. Elkashty, O.A.; Ashry, R.; Elghanam, G.A.; Pham, H.M.; Su, X.; Stegen, C.; Tran, S.D. Broccoli extract improves chemotherapeutic drug efficacy against head-neck squamous cell carcinomas. Med Oncol 2018, 35, https://doi.org/10.1007/s12032-018-1186-4. 
9. Kan, S.F.; Wang, J.; Sun, G.X. Sulforaphane regulates apoptosis- and proliferationrelated signaling pathways and synergizes with cisplatin to suppress human ovarian cancer. Int J Mol Med 2018, 42, 24472458, https://doi.org/10.3892/ijmm.2018.3860.

10. Singh, K.B.; Hahm, E.R.; Alumkal, J.J.; Foley, L.M.; Hitchens, T.K.; Shiva, S.S.; Parikh, R.A.; Jacobs, B.L.; Singh, S.V. Reversal of the Warburg phenomenon in chemoprevention of prostate cancer by sulforaphane. Carcinogenesis 2019, 40, 1545-1556, https://doi.org/10.1093/carcin/bgz155.

11. Bushati, N.; Cohen, S.M. MicroRNA functions. Annu Rev Cell Dev Bi 2007, 23, 175-205.

12. Lu, T.X.; Rothenberg, M.E. MicroRNA. J Allergy Clin Immunol 2018, 141, 1202-1207, https://doi.org/10.1016/j.jaci.2017.08.034.

13. Warde-Farley, D.; Donaldson, S.L.; Comes, O.; Zuberi, K.; Badrawi, R.; Chao, P.; Franz, M.; Grouios, C.; Kazi, F.; Lopes, C.T.; Maitland, A.; Mostafavi, S.; Montojo, J.; Shao, Q.; Wright, G.; Bader, G.D.; Morris, Q. The GeneMANIA prediction server: biological network integration for gene prioritization and predicting gene function. Nucleic Acids Research 2010, 38, W214-W220, https://doi.org/10.1093/nar/gkq537.

14. Kinser, H.E.; Pincus, Z. MicroRNAs as modulators of longevity and the aging process. Hum Genet 2020, 139, 291-308, https://doi.org/10.1007/s00439-019-02046-0.

15. Zhou, B.; Zuo, X.X.; Li, Y.S.; Gao, S.M.; Dai, X.D.; Zhu, H.L.; Luo, H. Integration of microRNA and mRNA expression profiles in the skin of systemic sclerosis patients. Sci Rep-Uk 2017, 7, https://doi.org/10.1038/srep42899.

16. Duttagupta, R.; Jiang, R.; Gollub, J.; Getts, R.C.; Jones, K.W. Impact of Cellular miRNAs on Circulating miRNA Biomarker Signatures. Plos One 2011, 6, https://doi.org/10.1371/journal.pone.0020769.

17. Gasparello, J.; Gambari, L.; Papi, C.; Rozzi, A.; Manicardi, A.; Corradini, R.; Gambari, R.; Finotti, A. High Levels of Apoptosis Are Induced in the Human Colon Cancer HT-29 Cell Line by Co-Administration of Sulforaphane and a Peptide Nucleic Acid Targeting miR-15b-5p. Nucleic Acid Ther 2020, 30, 164-174, https://doi.org/10.1089/nat.2019.0825.

18. Gao, L.; Cheng, D.; Yang, J.; Wu, R.; Li, W.; Kong, A.N. Sulforaphane epigenetically demethylates the CpG sites of the miR-9-3 promoter and reactivates miR-9-3 expression in human lung cancer A549 cells. J Nutr Biochem 2018, 56, 109-115, https://doi.org/10.1016/j.jnutbio.2018.01.015.

19. Kiani, S.; Akhavan-Niaki, H.; Fattahi, S.; Kavoosian, S.; Babaian Jelodar, N.; Bagheri, N.; Najafi Zarrini, H. Purified sulforaphane from broccoli (Brassica oleracea var. italica) leads to alterations of CDX1 and CDX2 expression and changes in miR-9 and miR-326 levels in human gastric cancer cells. Gene 2018, 678, 115-123, https://doi.org/10.1016/j.gene.2018.08.026.

20. Chen, X.; Jiang, Z.; Zhou, C.; Chen, K.; Li, X.; Wang, Z.; Wu, Z.; Ma, J.; Ma, Q.; Duan, W. Activation of Nrf2 by Sulforaphane Inhibits High Glucose-Induced Progression of Pancreatic Cancer via AMPK Dependent Signaling. Cell Physiol Biochem 2018, 50, 1201-1215, https://doi.org/10.1159/000494547.

21. Alyoussef, A.; Taha, M. Antitumor activity of sulforaphane in mice model of skin cancer via blocking sulfatase-2. Exp Dermatol 2019, 28, 28-34, https://doi.org/10.1111/exd.13802.

22. Koolivand, M.; Ansari, M.; Piroozian, F.; Moein, S.; Malek Zadeh, K. Alleviating the progression of acute myeloid leukemia (AML) by sulforaphane through controlling miR-155 levels. Mol Biol Rep 2018, 45, 24912499, https://doi.org/10.1007/s11033-018-4416-0.

23. Li, X.; Zhao, Z.; Li, M.; Liu, M.; Bahena, A.; Zhang, Y.; Zhang, Y.; Nambiar, C.; Liu, G. Sulforaphane promotes apoptosis, and inhibits proliferation and self-renewal of nasopharyngeal cancer cells by targeting STAT signal through miRNA-124-3p. Biomed Pharmacother 2018, 103, 473-481, https://doi.org/10.1016/j.biopha.2018.03.121.

24. Xia, Y.F.; Pei, G.H.; Wang, N.; Che, Y.C.; Yu, F.S.; Yin, F.F.; Liu, H.X.; Luo, B.; Wang, Y.K. miR-3156$3 \mathrm{p}$ is downregulated in HPV-positive cervical cancer and performs as a tumor-suppressive miRNA. Virol J 2017, 14, 20, https://doi.org/10.1186/s12985-017-0695-7.

25. Hua, F.F.; Liu, S.S.; Zhu, L.H.; Wang, Y.H.; Liang, X.; Ma, N.; Shi, H.R. MiRNA-338-3p regulates cervical cancer cells proliferation by targeting MACC1 through MAPK signaling pathway. Eur Rev Med Pharmacol Sci 2017, 21, 5342-5352, https://doi.org/10.26355/eurrev_201712_13919.

26. Bray, N.L.; Pimentel, H.; Melsted, P.; Pachter, L. Near-optimal probabilistic RNA-seq quantification. Nat Biotechnol 2016, 34, 525-527, https://doi.org/10.1038/nbt.3519.

27. Love, M.I.; Huber, W.; Anders, S. Moderated estimation of fold change and dispersion for RNA-seq data with DESeq2. Genome Biol 2014, 15, 550, https://doi.org/10.1186/s13059-014-0550-8.

28. Ai, C.; Kong, L. CGPS: A machine learning-based approach integrating multiple gene set analysis tools for better prioritization of biologically relevant pathways. J Genet Genomics 2018, 45, 489-504, https://doi.org/10.1016/j.jgg.2018.08.002.

29. Friedlander, M.R.; Mackowiak, S.D.; Li, N.; Chen, W.; Rajewsky, N. miRDeep2 accurately identifies known and hundreds of novel microRNA genes in seven animal clades. Nucleic Acids Res 2012, 40, 37-52, https://doi.org/10.1093/nar/gkr688.

30. Kozomara, A.; Birgaoanu, M.; Griffiths-Jones, S. miRBase: from microRNA sequences to function. Nucleic Acids Res 2019, 47, D155-D162, https://doi.org/10.1093/nar/gky1141.

31. Langmead, B.; Salzberg, S.L. Fast gapped-read alignment with Bowtie 2. Nat Methods 2012, 9, 357-359, https://doi.org/10.1038/nmeth.1923. 
32. Agarwal, V.; Bell, G.W.; Nam, J.W.; Bartel, D.P. Predicting effective microRNA target sites in mammalian mRNAs. Elife 2015, 4, https://doi.org/10.7554/eLife.05005.

33. Goan, Y.G.; Wu, W.T.; Liu, C.I.; Neoh, C.A.; Wu, Y.J. Involvement of Mitochondrial Dysfunction, Endoplasmic Reticulum Stress, and the PI3K/AKT/mTOR Pathway in Nobiletin-Induced Apoptosis of Human Bladder Cancer Cells. Molecules 2019, 24, https://doi.org/10.3390/molecules24162881.

34. Lan, F.; Pan, Q.; Yu, H.; Yue, X. Sulforaphane enhances temozolomide-induced apoptosis because of downregulation of miR-21 via Wnt/beta-catenin signaling in glioblastoma. J Neurochem 2015, 134, 811-818, https://doi.org/10.1111/jnc.13174.

35. Eren, E.; Tufekci, K.U.; Isci, K.B.; Tastan, B.; Genc, K.; Genc, S. Sulforaphane Inhibits LipopolysaccharideInduced Inflammation, Cytotoxicity, Oxidative Stress, and miR-155 Expression and Switches to Mox Phenotype through Activating Extracellular Signal-Regulated Kinase 1/2-Nuclear Factor Erythroid 2Related Factor 2/Antioxidant Response Element Pathway in Murine Microglial Cells. Front Immunol 2018, 9, https://doi.org/10.3389/fimmu.2018.00036.

36. An, Y.W.; Jhang, K.A.; Woo, S.Y.; Kang, J.L.; Chong, Y.H. Sulforaphane exerts its anti-inflammatory effect against amyloid-beta peptide via STAT-1 dephosphorylation and activation of Nrf2/HO-1 cascade in human THP-1 macrophages. $\quad$ Neurobiol Aging $\quad \mathbf{2 0 1 6 ,} \quad 38, \quad$ 1-10, https://doi.org/10.1016/j.neurobiolaging.2015.10.016.

37. Wang, D.X.; Zou, Y.J.; Zhuang, X.B.; Chen, S.X.; Lin, Y.; Li, W.L.; Lin, J.J.; Lin, Z.Q. Sulforaphane suppresses EMT and metastasis in human lung cancer through miR-616-5p-mediated GSK3beta/betacatenin signaling pathways. Acta Pharmacol Sin 2017, 38, 241-251, https://doi.org/10.1038/aps.2016.122.

38. Reddy, S.A.; Shelar, S.B.; Dang, T.M.; Lee, B.N.; Yang, H.; Ong, S.M.; Ng, H.L.; Chui, W.K.; Wong, S.C.; Chew, E.H. Sulforaphane and its methylcarbonyl analogs inhibit the LPS-stimulated inflammatory response in human monocytes through modulating cytokine production, suppressing chemotactic migration and phagocytosis in a NF-kappaB- and MAPK-dependent manner. Int Immunopharmacol 2015, 24, 440-450, https://doi.org/10.1016/j.intimp.2014.12.037.

39. Shan, Y.; Wang, X.; Wang, W.; He, C.; Bao, Y. p38 MAPK plays a distinct role in sulforaphane-induced up-regulation of ARE-dependent enzymes and down-regulation of COX-2 in human bladder cancer cells. Oncol Rep 2010, 23, 1133-1138, https://doi.org/10.3892/or_00000742.

40. Ren, J.; Yuan, L.; Wang, Y.; Chen, G.; Hu, K. Benzyl sulforaphane is superior to sulforaphane in inhibiting the Akt/MAPK and activating the Nrf2/ARE signalling pathways in HepG2 cells. J Pharm Pharmacol 2018, 70, 1643-1653, https://doi.org/10.1111/jphp.13015.

41. Qin, S.; Yang, C.; Huang, W.; Du, S.; Mai, H.; Xiao, J.; Lu, T. Sulforaphane attenuates microglia-mediated neuronal necroptosis through down-regulation of MAPK/NF-kappaB signaling pathways in LPS-activated BV-2 microglia. Pharmacol Res 2018, 133, 218-235, https://doi.org/10.1016/j.phrs.2018.01.014.

42. Mondal, A.; Biswas, R.; Rhee, Y.H.; Kim, J.; Ahn, J.C. Sulforaphene promotes Bax/Bcl2, MAPK-dependent human gastric cancer AGS cells apoptosis and inhibits migration via EGFR, p-ERK1/2 down-regulation. Gen Physiol Biophys 2016, 35, 25-34.

43. Kim, B.G.; Fujita, T.; Stankovic, K.M.; Welling, D.B.; Moon, I.S.; Choi, J.Y.; Yun, J.; Kang, J.S.; Lee, J.D. Sulforaphane, a natural component of broccoli, inhibits vestibular schwannoma growth in vitro and in vivo. Sci Rep 2016, 6, 36215, https://doi.org/10.1038/srep36215. 
Supplementary files

\section{SupplyTable 1}

\begin{tabular}{|c|}
\hline Target \\
\hline BOK \\
\hline CXCR3 \\
\hline SHOX \\
\hline CRX \\
\hline KCTD17 \\
\hline SLC25A34 \\
\hline DLX2 \\
\hline C1QTNF6 \\
\hline SELM \\
\hline PNMA6C \\
\hline PNMA6A \\
\hline SMIM7 \\
\hline AL590822.2 \\
\hline LTBP4 \\
\hline AGXT \\
\hline PHLDB3 \\
\hline C17orf103 \\
\hline GNLY \\
\hline ALKBH4 \\
\hline TMUB1 \\
\hline TECPR1 \\
\hline CCL17 \\
\hline SPEF1 \\
\hline TOR4A \\
\hline AC115618.1 \\
\hline FOXA3 \\
\hline B4GALT3 \\
\hline CEP72 \\
\hline NDP \\
\hline SERPINF2 \\
\hline C15orf62 \\
\hline ORAI2 \\
\hline PTPRN2 \\
\hline IL25 \\
\hline TREML1 \\
\hline LUC7L \\
\hline FLJ00104 \\
\hline AC025278.1 \\
\hline TRPV2 \\
\hline RP11-863K10.7 \\
\hline TUBB2B \\
\hline GNB1L \\
\hline ARHGDIG \\
\hline ANKS6 \\
\hline ZMAT3 \\
\hline PTGES \\
\hline GOSR2 \\
\hline MAP4 \\
\hline AC000003.2 \\
\hline PLCXD1 \\
\hline CTXN1 \\
\hline CACNG4 \\
\hline UTS2R \\
\hline BTBD19 \\
\hline PNMA3 \\
\hline C13orf35 \\
\hline
\end{tabular}

\begin{tabular}{|c|}
\hline SFRP5 \\
\hline TBXA2R \\
\hline HMBS \\
\hline KIAA0513 \\
\hline MEX3B \\
\hline PRX \\
\hline MARVELD1 \\
\hline AOAH \\
\hline HSH2D \\
\hline SYVN1 \\
\hline SLC39A3 \\
\hline LY6G5B \\
\hline TUSC5 \\
\hline IL31 \\
\hline $\mathrm{HIC1}$ \\
\hline PSME3 \\
\hline TCL1A \\
\hline REM1 \\
\hline OTUD6A \\
\hline TSSK6 \\
\hline RNF125 \\
\hline C17orf107 \\
\hline C10orf105 \\
\hline IER5L \\
\hline C3orf80 \\
\hline STX4 \\
\hline CASP16 \\
\hline USP11 \\
\hline PTGES2 \\
\hline MGAT5B \\
\hline STAT5A \\
\hline VPS37B \\
\hline PPP1R27 \\
\hline LHFPL5 \\
\hline SCNN1G \\
\hline CSNK2B-LY6G5B-1181 \\
\hline DDX11 \\
\hline TMEM95 \\
\hline NEU1 \\
\hline IL23R \\
\hline DIDO1 \\
\hline DZANK1 \\
\hline RP11-834C11.12 \\
\hline PDDC1 \\
\hline AC006967.1 \\
\hline GPR108 \\
\hline C19orf25 \\
\hline SYT5 \\
\hline HAGHL \\
\hline SMPD3 \\
\hline POU3F1 \\
\hline CTD-2054N24.2 \\
\hline ALDH1A3 \\
\hline GAB4 \\
\hline GJD3 \\
\hline VAV1 \\
\hline SIX2 \\
\hline
\end{tabular}

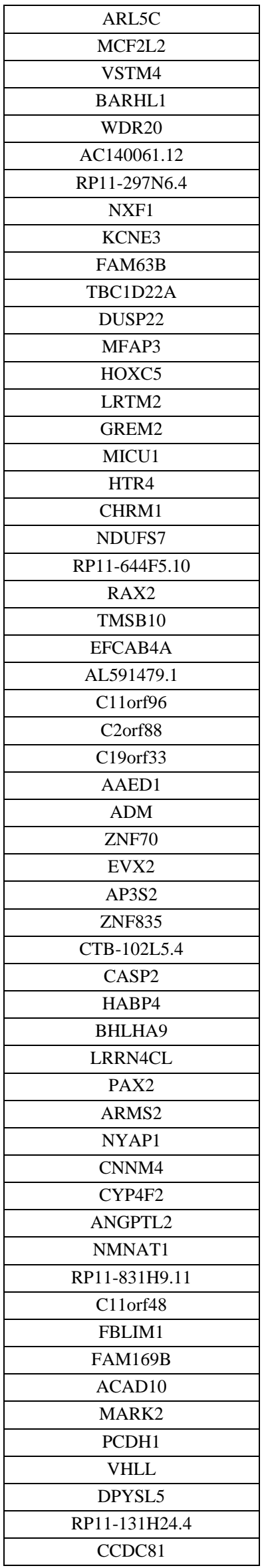


https://doi.org/10.33263/BRIAC111.79437972

\begin{tabular}{|c|}
\hline ABHD17B \\
\hline GDPD1 \\
\hline CHST13 \\
\hline C6orf226 \\
\hline INPP5D \\
\hline CARD14 \\
\hline WBSCR16 \\
\hline TXNRD1 \\
\hline LRRC1 \\
\hline GPR75 \\
\hline SCARF1 \\
\hline ENGASE \\
\hline ADRB1 \\
\hline LRFN1 \\
\hline AGPAT2 \\
\hline MDM4 \\
\hline PARP10 \\
\hline RASD2 \\
\hline STAT1 \\
\hline KCNK13 \\
\hline SFTPC \\
\hline AJAP1 \\
\hline ZNF454 \\
\hline LCNL1 \\
\hline POU4F1 \\
\hline ULK3 \\
\hline C15orf38-AP3S2 \\
\hline B3GAT3 \\
\hline WFDC8 \\
\hline PSAPL1 \\
\hline KLHL17 \\
\hline L2HGDH \\
\hline SLC6A1 \\
\hline SLC30A7 \\
\hline FOXD2 \\
\hline ATXN7L3 \\
\hline DHRS4 \\
\hline FAM84A \\
\hline ZNF430 \\
\hline RASA3 \\
\hline GLRA4 \\
\hline ZBTB8A \\
\hline IFITM5 \\
\hline $\mathrm{CPZ}$ \\
\hline CLPS \\
\hline TIMM44 \\
\hline IVD \\
\hline PRKAB1 \\
\hline KCNIP3 \\
\hline NFIX \\
\hline MDM2 \\
\hline TXNRD2 \\
\hline MTRNR2L4 \\
\hline SLC52A3 \\
\hline ZNF234 \\
\hline AC009892.10 \\
\hline USP36 \\
\hline FOXK1 \\
\hline MRPL55 \\
\hline CELF5 \\
\hline GABPB2 \\
\hline PTK6 \\
\hline
\end{tabular}

\begin{tabular}{|c|}
\hline CDKL1 \\
\hline A4GALT \\
\hline TLE6 \\
\hline AC021860.1 \\
\hline MIF \\
\hline CRKL \\
\hline ZNF154 \\
\hline CXorf56 \\
\hline CTRC \\
\hline KIF14 \\
\hline ZFPL1 \\
\hline OVGP1 \\
\hline TPM1 \\
\hline XPNPEP2 \\
\hline SGSM1 \\
\hline OAZ3 \\
\hline GSG2 \\
\hline CREB3L3 \\
\hline C14orf182 \\
\hline AC140481.2 \\
\hline MRFAP1 \\
\hline PPARD \\
\hline MFI2 \\
\hline IGHMBP2 \\
\hline PAOX \\
\hline ZNF106 \\
\hline TRIM8 \\
\hline Z98049.1 \\
\hline ZNF791 \\
\hline LRRC3DN \\
\hline SP140L \\
\hline FOXC2 \\
\hline CHST6 \\
\hline CD209 \\
\hline SOCS2 \\
\hline FAM9B \\
\hline GTF2IRD1 \\
\hline MRPL28 \\
\hline MOGS \\
\hline SYK \\
\hline TBC1D28 \\
\hline HCLS1 \\
\hline C1orf159 \\
\hline RRP7A \\
\hline AP005482.1 \\
\hline PSMG4 \\
\hline EIF4EBP2 \\
\hline ALPI \\
\hline NR1I3 \\
\hline TAF8 \\
\hline OGG1 \\
\hline SOWAHD \\
\hline AMER3 \\
\hline CPT1A \\
\hline FADS6 \\
\hline RP11-93B14.6 \\
\hline AFMID \\
\hline GRK1 \\
\hline DIRAS1 \\
\hline VWA1 \\
\hline TACSTD2 \\
\hline CBX6 \\
\hline
\end{tabular}

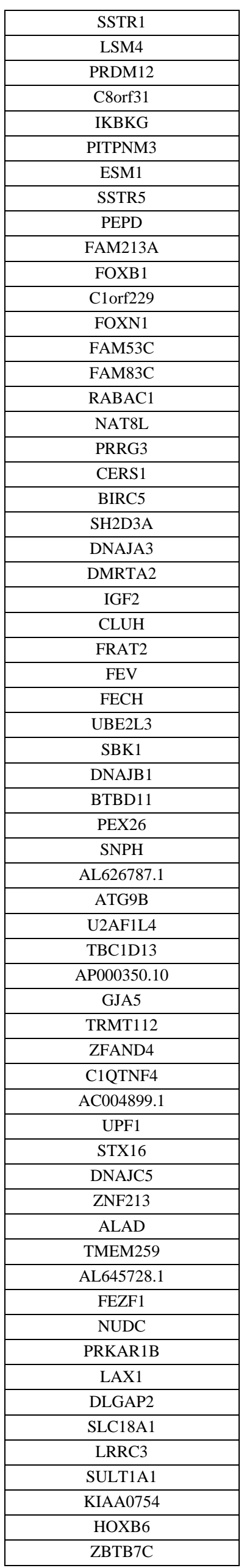

https://biointerfaceresearch.com/ 
https://doi.org/10.33263/BRIAC111.79437972

\begin{tabular}{|c|}
\hline COL4A5 \\
\hline AC006486.1 \\
\hline SLC39A11 \\
\hline POM121L7 \\
\hline SMARCD3 \\
\hline SYT15 \\
\hline CCM2L \\
\hline TMEM107 \\
\hline AL359091.2 \\
\hline GATA2 \\
\hline STPG1 \\
\hline ELF3 \\
\hline SNCB \\
\hline NTSR1 \\
\hline KCNAB3 \\
\hline ARIH2OS \\
\hline NWD1 \\
\hline RTN4RL1 \\
\hline TARS2 \\
\hline MED6 \\
\hline AC005003.1 \\
\hline FBXL18 \\
\hline HAGH \\
\hline AL020996.1 \\
\hline TMEM145 \\
\hline NLRP12 \\
\hline LINC00908 \\
\hline FAHD1 \\
\hline MLC1 \\
\hline TEN1 \\
\hline FAIM2 \\
\hline ING5 \\
\hline MPV17L \\
\hline MUL1 \\
\hline SIGLEC11 \\
\hline C14orf23 \\
\hline MCAT \\
\hline KCNH6 \\
\hline GNB2 \\
\hline RNASEK \\
\hline C2orf48 \\
\hline SLC44A4 \\
\hline VMO1 \\
\hline CCL5 \\
\hline PLEKHA4 \\
\hline TPD52L2 \\
\hline TSPO \\
\hline CDK10 \\
\hline MAGEB10 \\
\hline CTB-167G5.5 \\
\hline PPID \\
\hline AL357673.1 \\
\hline CTA-299D3.8 \\
\hline CASP3 \\
\hline DPH7 \\
\hline NTRK2 \\
\hline MTHFSD \\
\hline ADCK4 \\
\hline ROGDI \\
\hline APCDD1L \\
\hline SNAI1 \\
\hline AC022532.1 \\
\hline
\end{tabular}

\begin{tabular}{|c|}
\hline WNT7B \\
\hline TNFSF10 \\
\hline CTSD \\
\hline C12orf68 \\
\hline FAM60A \\
\hline DAND5 \\
\hline CALML6 \\
\hline FAM76A \\
\hline TOMM40 \\
\hline C5AR2 \\
\hline AC016722.1 \\
\hline TMEM132E \\
\hline CPLX2 \\
\hline LYZ \\
\hline SYNDIG1 \\
\hline CXCL14 \\
\hline HM13 \\
\hline FAM86A \\
\hline CACNB1 \\
\hline RCAN1 \\
\hline KALRN \\
\hline GINS4 \\
\hline NCR3LG1 \\
\hline FAM110D \\
\hline BLOC1S3 \\
\hline STAU1 \\
\hline APOBEC3A \\
\hline CEACAM5 \\
\hline CD300E \\
\hline OSCAR \\
\hline CAMK1D \\
\hline METTL8 \\
\hline FAM78A \\
\hline SPTBN2 \\
\hline PIGW \\
\hline NKX6-3 \\
\hline CYB561 \\
\hline OSBPL2 \\
\hline FAM156B \\
\hline SCRT1 \\
\hline FAM156A \\
\hline CHMP6 \\
\hline RPH3AL \\
\hline SLC39A13 \\
\hline SLC9A1 \\
\hline ALDH18A1 \\
\hline CDC14B \\
\hline DNAJC14 \\
\hline GPR35 \\
\hline ARHGAP19-SLIT1 \\
\hline SCN5A \\
\hline C5orf45 \\
\hline ADAMTS4 \\
\hline COPG1 \\
\hline SLC14A2 \\
\hline HIST1H2AG \\
\hline ZNF175 \\
\hline C4orf26 \\
\hline ZNF157 \\
\hline HPCAL1 \\
\hline ZGLP1 \\
\hline NEK8 \\
\hline
\end{tabular}

\begin{tabular}{|c|}
\hline CELF6 \\
\hline NUBP2 \\
\hline XYLT2 \\
\hline EN1 \\
\hline AMBP \\
\hline SERF1A \\
\hline SERF1B \\
\hline AC016559.1 \\
\hline FANCC \\
\hline DAB2IP \\
\hline GGA3 \\
\hline DDX51 \\
\hline SCRT2 \\
\hline FCRLB \\
\hline ARSK \\
\hline PIM1 \\
\hline CD300C \\
\hline ZBTB47 \\
\hline URAD \\
\hline CHRNB1 \\
\hline GLTP \\
\hline RAB40C \\
\hline WIZ \\
\hline CEACAM18 \\
\hline GDPD5 \\
\hline KANK2 \\
\hline SFMBT2 \\
\hline SLC35E3 \\
\hline NT5C1A \\
\hline NRTN \\
\hline RBM7 \\
\hline $\mathrm{CIC}$ \\
\hline C19orf40 \\
\hline RADIL \\
\hline MX1 \\
\hline TNNT2 \\
\hline CARD10 \\
\hline SERBP1 \\
\hline AC105020.1 \\
\hline NHSL1 \\
\hline LRRC27 \\
\hline ST8SIA2 \\
\hline RBM3 \\
\hline ANKRD40 \\
\hline TRPM2 \\
\hline EPN3 \\
\hline FAM64A \\
\hline LOH12CR2 \\
\hline NCS1 \\
\hline AC079210.1 \\
\hline ALDH3B1 \\
\hline ENSG00000270466 \\
\hline SGTA \\
\hline NSMF \\
\hline ZNF773 \\
\hline PHYHD1 \\
\hline NKX2-5 \\
\hline NAT9 \\
\hline RPS15A \\
\hline PAK4 \\
\hline WFDC1 \\
\hline NDNL2 \\
\hline
\end{tabular}

https://biointerfaceresearch.com/ 
https://doi.org/10.33263/BRIAC111.79437972

\begin{tabular}{|c|}
\hline FDX1L \\
\hline ENTPD6 \\
\hline NELFB \\
\hline RSL1D1 \\
\hline TRAPPC2 \\
\hline TRIM54 \\
\hline WIPI1 \\
\hline FGFR4 \\
\hline PPP1R3G \\
\hline PIPOX \\
\hline KIAA1257 \\
\hline PPP1R8 \\
\hline CARM1 \\
\hline NAALADL1 \\
\hline ICAM4 \\
\hline MBOAT1 \\
\hline NR1H2 \\
\hline PRKX \\
\hline EXTL1 \\
\hline FFAR2 \\
\hline LGI3 \\
\hline LAIR1 \\
\hline UBE2G2 \\
\hline TLR10 \\
\hline APOL2 \\
\hline PRDM8 \\
\hline TAF13 \\
\hline LRRC14B \\
\hline EFNA2 \\
\hline FLJ30594 \\
\hline COX7B \\
\hline LYRM2 \\
\hline NFIC \\
\hline DUX4L2 \\
\hline TUBB6 \\
\hline OSM \\
\hline P4HB \\
\hline ELMOD1 \\
\hline ARF5 \\
\hline AC112693.2 \\
\hline ANO10 \\
\hline CRCP \\
\hline TMED4 \\
\hline ADRBK1 \\
\hline ARL6IP1 \\
\hline APITD1-CORT \\
\hline C9orf66 \\
\hline CTD-2162K18.4 \\
\hline INPPL1 \\
\hline ASCL5 \\
\hline ANKRD36 \\
\hline GJA9 \\
\hline NICN1 \\
\hline ZFP42 \\
\hline TUBA3D \\
\hline ID3 \\
\hline ZBP1 \\
\hline GTPBP3 \\
\hline CTD-2368P22.1 \\
\hline TWF2 \\
\hline FAM86B1 \\
\hline DDX31 \\
\hline
\end{tabular}

\begin{tabular}{|c|}
\hline ATP13A1 \\
\hline NRXN2 \\
\hline METTL7A \\
\hline NBEAL2 \\
\hline TNFRSF1B \\
\hline KCTD5 \\
\hline RETSAT \\
\hline KLHL30 \\
\hline SOBP \\
\hline TMCO3 \\
\hline ATP6V1B1 \\
\hline FBLN5 \\
\hline C7orf43 \\
\hline MYO3A \\
\hline MKNK2 \\
\hline SLC2A6 \\
\hline P2RX7 \\
\hline H1F0 \\
\hline NRIP3 \\
\hline CYTH4 \\
\hline C10orf55 \\
\hline TNFSF14 \\
\hline CDK5R2 \\
\hline C2orf15 \\
\hline MRPL30 \\
\hline CASC10 \\
\hline COQ10B \\
\hline SPC24 \\
\hline SYNGR1 \\
\hline SNRPB \\
\hline TBC1D32 \\
\hline PTX4 \\
\hline HAND1 \\
\hline TBCCD1 \\
\hline TAF1 \\
\hline RAB8A \\
\hline RRP1B \\
\hline IFFO1 \\
\hline UBXN2A \\
\hline MLX \\
\hline ECSIT \\
\hline ZNF143 \\
\hline LYSMD1 \\
\hline CA13 \\
\hline IL10RB \\
\hline TAB1 \\
\hline INPP5E \\
\hline NANOG \\
\hline FRMD1 \\
\hline FAM219A \\
\hline EFHD2 \\
\hline RICTOR \\
\hline QRFPR \\
\hline ATPIF1 \\
\hline NTM \\
\hline LPCAT2 \\
\hline UST \\
\hline IL21R \\
\hline TCF7 \\
\hline BAIAP2L1 \\
\hline ZNF512B \\
\hline ARMC5 \\
\hline
\end{tabular}

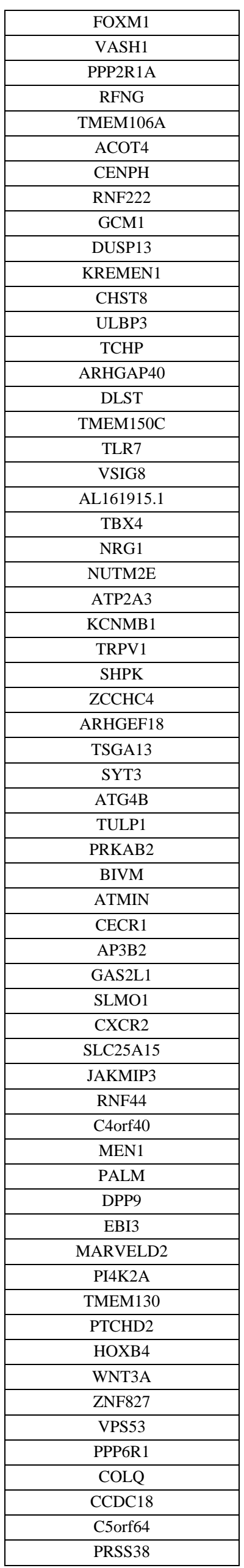

https://biointerfaceresearch.com/ 
https://doi.org/10.33263/BRIAC111.79437972

\begin{tabular}{|c|}
\hline XKR8 \\
\hline ALG1 \\
\hline AL450307.1 \\
\hline MLLT4 \\
\hline CIB2 \\
\hline RIOK3 \\
\hline KBTBD6 \\
\hline PIM2 \\
\hline SLC7A14 \\
\hline POLH \\
\hline AKT1S1 \\
\hline C8orf82 \\
\hline TOR2A \\
\hline USP24 \\
\hline RNF224 \\
\hline RWDD2A \\
\hline ACOT9 \\
\hline SLC2A3 \\
\hline VGF \\
\hline SPDEF \\
\hline FAM53A \\
\hline EVC \\
\hline CTDSP2 \\
\hline PARD6G \\
\hline SIRT6 \\
\hline TGIF2 \\
\hline HMG20A \\
\hline GRK7 \\
\hline MAPK8IP3 \\
\hline CYP51A1 \\
\hline ZNF383 \\
\hline PSTPIP2 \\
\hline PRRG2 \\
\hline TECPR2 \\
\hline RP11-1118M6.1 \\
\hline FLNB \\
\hline AC137932.1 \\
\hline RAET1E \\
\hline LMOD3 \\
\hline AC084121.16 \\
\hline MS4A7 \\
\hline PYGB \\
\hline MRTO4 \\
\hline BPNT1 \\
\hline AC104841.2 \\
\hline KRT15 \\
\hline ECT2L \\
\hline C1orf111 \\
\hline KCNF1 \\
\hline DUSP18 \\
\hline TSNARE1 \\
\hline TAF1D \\
\hline RAB3B \\
\hline STK40 \\
\hline CD82 \\
\hline MYO3B \\
\hline ZNF362 \\
\hline CALM3 \\
\hline MINOS1 \\
\hline CLSTN1 \\
\hline NHLH1 \\
\hline SYNE2 \\
\hline
\end{tabular}

\begin{tabular}{|c|}
\hline H6PD \\
\hline LCN6 \\
\hline ZBTB8OS \\
\hline ICOSLG \\
\hline TNFAIP3 \\
\hline HIST2H3C \\
\hline NCK2 \\
\hline HIST2H3A \\
\hline MYO1C \\
\hline ADM2 \\
\hline GDNF \\
\hline SNAPC2 \\
\hline TMEM105 \\
\hline ZNF697 \\
\hline PODXL2 \\
\hline HSPA6 \\
\hline ZNF556 \\
\hline RAB3D \\
\hline ABCB8 \\
\hline HIST1H2BF \\
\hline BSG \\
\hline C21orf2 \\
\hline FAM20C \\
\hline ARFRP1 \\
\hline WDR92 \\
\hline ZBTB39 \\
\hline RINL \\
\hline C19orf35 \\
\hline SCAMP5 \\
\hline ZNF492 \\
\hline ZNF44 \\
\hline SLC16A13 \\
\hline MAVS \\
\hline POU4F3 \\
\hline ZNF91 \\
\hline STAC2 \\
\hline DAPP1 \\
\hline SLC25A32 \\
\hline PARD6B \\
\hline FBXL22 \\
\hline EXD1 \\
\hline PIWIL2 \\
\hline KCNT1 \\
\hline 1-Mar \\
\hline PDE6G \\
\hline OR7D2 \\
\hline PDE4C \\
\hline AC093157.1 \\
\hline KRT6B \\
\hline SLC29A4 \\
\hline HCAR1 \\
\hline C2orf50 \\
\hline WDR5B \\
\hline MOCOS \\
\hline ANKRD29 \\
\hline THAP5 \\
\hline PARD3B \\
\hline HAPLN4 \\
\hline CASKIN1 \\
\hline VHL \\
\hline ECE1 \\
\hline GCAT \\
\hline
\end{tabular}

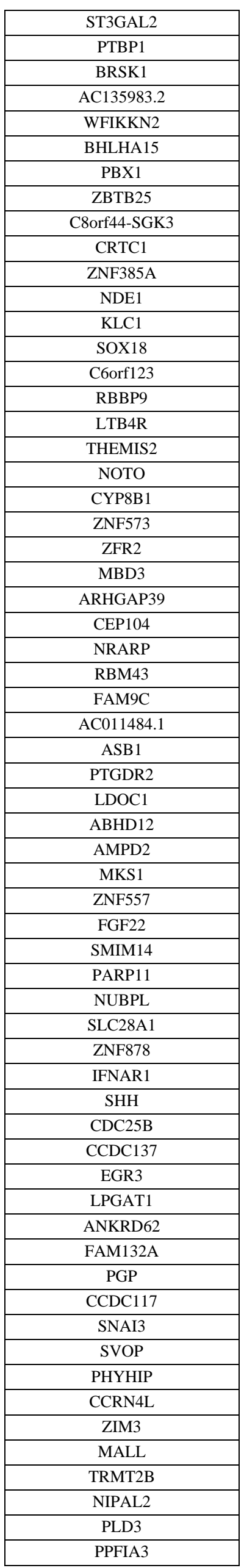

https://biointerfaceresearch.com/ 
https://doi.org/10.33263/BRIAC111.79437972

\begin{tabular}{|c|}
\hline ZNF665 \\
\hline HTR1D \\
\hline RD3 \\
\hline TP53 \\
\hline PHF20 \\
\hline STK11 \\
\hline TSPYL1 \\
\hline DTX3L \\
\hline POM121 \\
\hline WBP2 \\
\hline ZFYVE26 \\
\hline SSBP3 \\
\hline CX3CL1 \\
\hline TAF1L \\
\hline NR2F6 \\
\hline SPI1 \\
\hline ESCO2 \\
\hline PMM2 \\
\hline RBM48 \\
\hline ARSD \\
\hline LL22NC03-63E9.3 \\
\hline ZNF587B \\
\hline SCARA3 \\
\hline SGK3 \\
\hline HRH1 \\
\hline SOST \\
\hline VWA3A \\
\hline AC145676.2 \\
\hline KCNJ15 \\
\hline C21orf90 \\
\hline PSMB11 \\
\hline YPEL2 \\
\hline NOS1AP \\
\hline TNFAIP2 \\
\hline ANKRD63 \\
\hline PHF21B \\
\hline MFSD7 \\
\hline NAPA \\
\hline NUAK2 \\
\hline PAICS \\
\hline POLR2D \\
\hline RPH3A \\
\hline ATAD3C \\
\hline RPS21 \\
\hline TLCD2 \\
\hline HRH4 \\
\hline TNK2 \\
\hline LAMP2 \\
\hline OTOF \\
\hline AC079612.1 \\
\hline KCNK6 \\
\hline TRAFD1 \\
\hline ZNF813 \\
\hline ACVRL1 \\
\hline KCNK5 \\
\hline OLFML2A \\
\hline ALG12 \\
\hline GPR82 \\
\hline GSX1 \\
\hline SLC35B4 \\
\hline GPR176 \\
\hline ZNF528 \\
\hline
\end{tabular}

\begin{tabular}{|c|}
\hline GINM1 \\
\hline RIPPLY3 \\
\hline NFATC2IP \\
\hline VIPR1 \\
\hline DUX4L5 \\
\hline C22orf46 \\
\hline FOXJ3 \\
\hline MAPK14 \\
\hline GATC \\
\hline FAM173A \\
\hline SNX22 \\
\hline LAMP3 \\
\hline SUN2 \\
\hline SLC26A4 \\
\hline TNNT3 \\
\hline RGS9BP \\
\hline KEAP1 \\
\hline RDH13 \\
\hline CLEC16A \\
\hline FAM19A5 \\
\hline SEMA7A \\
\hline PDLIM5 \\
\hline CES4A \\
\hline GJB1 \\
\hline FUT1 \\
\hline ERAP2 \\
\hline FOXQ1 \\
\hline ZNF101 \\
\hline AQP2 \\
\hline OPA3 \\
\hline POU2F2 \\
\hline DUX4 \\
\hline MYO16 \\
\hline DUX4L3 \\
\hline DUX4L7 \\
\hline DUX4L4 \\
\hline LHX3 \\
\hline ITGAX \\
\hline DUX4L6 \\
\hline ADCY9 \\
\hline MYOD1 \\
\hline LILRB3 \\
\hline FAM217B \\
\hline EREG \\
\hline RFX1 \\
\hline PRR26 \\
\hline USP54 \\
\hline ZNF793 \\
\hline VEGFA \\
\hline MBP \\
\hline INMT \\
\hline C6orf223 \\
\hline SURF6 \\
\hline PITX1 \\
\hline AC006372.1 \\
\hline HLA-E \\
\hline MPL \\
\hline KRT86 \\
\hline $\mathrm{CDH} 4$ \\
\hline ARX \\
\hline AL021546.6 \\
\hline DOK7 \\
\hline
\end{tabular}

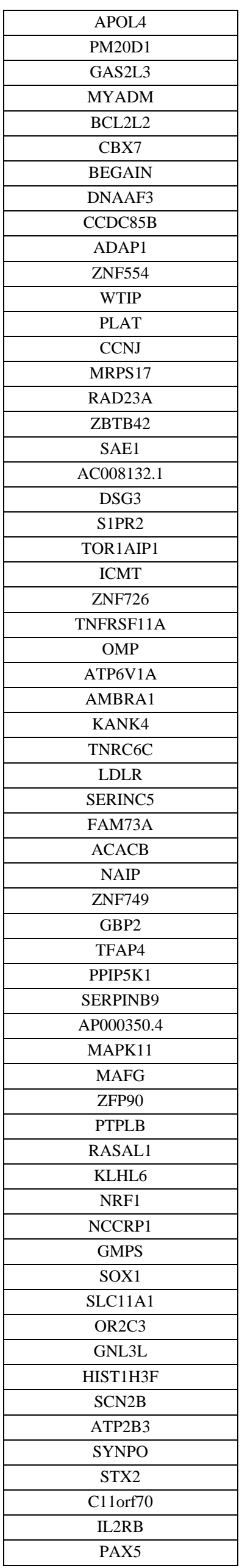

https://biointerfaceresearch.com/ 
https://doi.org/10.33263/BRIAC111.79437972

\begin{tabular}{|c|}
\hline SCARF2 \\
\hline RNF34 \\
\hline CASP8 \\
\hline CRISPLD2 \\
\hline IGFN1 \\
\hline 9-Sep \\
\hline IRF5 \\
\hline CA12 \\
\hline FAM213B \\
\hline SMARCD1 \\
\hline SCD5 \\
\hline PGBD4 \\
\hline PHF7 \\
\hline CCDC40 \\
\hline NUDT4 \\
\hline PITPNM2 \\
\hline GMEB2 \\
\hline ISLR2 \\
\hline SLC46A1 \\
\hline NAGPA \\
\hline ACVR1B \\
\hline OTUD3 \\
\hline ANKS3 \\
\hline HIF1AN \\
\hline DENR \\
\hline FKTN \\
\hline TCF21 \\
\hline FGD2 \\
\hline HSPB6 \\
\hline UNC5B \\
\hline PIAS4 \\
\hline HRAS \\
\hline DCAF16 \\
\hline DHDDS \\
\hline ZNF2 \\
\hline DCP1A \\
\hline TMEM236 \\
\hline TMEM236 \\
\hline SLC7A6 \\
\hline SLIT1 \\
\hline IL17RE \\
\hline MFSD2B \\
\hline FUT3 \\
\hline FZD7 \\
\hline SFXN5 \\
\hline ZNF418 \\
\hline OTUD5 \\
\hline FICD \\
\hline COL27A1 \\
\hline MFN1 \\
\hline SPRYD3 \\
\hline TAL2 \\
\hline NCLN \\
\hline RPL36 \\
\hline MCMDC2 \\
\hline ACSS1 \\
\hline TSTA3 \\
\hline RPS6KB1 \\
\hline STK32A \\
\hline PRKAA1 \\
\hline IKZF4 \\
\hline ACTRT3 \\
\hline
\end{tabular}

\begin{tabular}{|c|}
\hline PSMB2 \\
\hline LIPH \\
\hline WDR55 \\
\hline NPAS4 \\
\hline $\mathrm{KCNC1}$ \\
\hline GLTPD1 \\
\hline ZSCAN22 \\
\hline SP1 \\
\hline PHKA2 \\
\hline MGAT3 \\
\hline GNE \\
\hline TRIM35 \\
\hline UBN2 \\
\hline KIAA1147 \\
\hline ABCF3 \\
\hline NUP155 \\
\hline CST3 \\
\hline C14orf132 \\
\hline FKBP14 \\
\hline RNF4 \\
\hline THAP6 \\
\hline MCC \\
\hline FSD2 \\
\hline CACNA1C \\
\hline KIAA1919 \\
\hline ITGB3 \\
\hline BRCA1 \\
\hline GPR116 \\
\hline TEF \\
\hline FLG2 \\
\hline MINOS1-NBL1 \\
\hline PRELP \\
\hline SRRM4 \\
\hline GPR144 \\
\hline ZNF695 \\
\hline ZNF397 \\
\hline EFNA3 \\
\hline MTL5 \\
\hline ICA1L \\
\hline MCCC2 \\
\hline FEM1A \\
\hline ZNF618 \\
\hline NKX3-2 \\
\hline CTSF \\
\hline TERF2 \\
\hline MAP2K4 \\
\hline UTP11L \\
\hline GRINA \\
\hline KNOP1 \\
\hline SYTL4 \\
\hline ZNF543 \\
\hline TAS2R5 \\
\hline ARMC10 \\
\hline$\overline{\text { CASZ1 }}$ \\
\hline ZNF669 \\
\hline LPIN3 \\
\hline NR1I2 \\
\hline FAM83H \\
\hline IL11 \\
\hline ARID3A \\
\hline SPPL2A \\
\hline GRIN3A \\
\hline
\end{tabular}

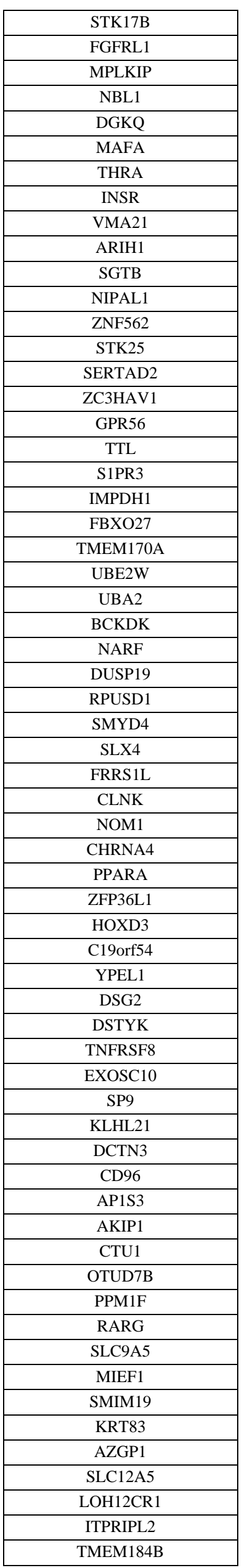

https://biointerfaceresearch.com/ 
https://doi.org/10.33263/BRIAC111.79437972

\begin{tabular}{|c|}
\hline SLC12A3 \\
\hline GPR123 \\
\hline KIAA0930 \\
\hline SPOCD1 \\
\hline SPN \\
\hline ASB16 \\
\hline PHACTR2 \\
\hline B3GALNT2 \\
\hline CHL1 \\
\hline LRRC10B \\
\hline KIAA1614 \\
\hline DENND6B \\
\hline ZNF487 \\
\hline SP8 \\
\hline TAPBP \\
\hline AC019294.1 \\
\hline LLGL2 \\
\hline AIRE \\
\hline TXNDC15 \\
\hline SP110 \\
\hline OCLN \\
\hline WWP2 \\
\hline ITPK1 \\
\hline ELOVL3 \\
\hline PACSIN2 \\
\hline FAM227A \\
\hline LRRC58 \\
\hline YIPF4 \\
\hline GIT2 \\
\hline FBXO46 \\
\hline CLSPN \\
\hline FSCN1 \\
\hline SFRP1 \\
\hline POM121C \\
\hline APLN \\
\hline RNF144A \\
\hline C1orf170 \\
\hline MR1 \\
\hline TMEM127 \\
\hline LRP10 \\
\hline AGMAT \\
\hline ZNF740 \\
\hline GNB5 \\
\hline BRI3BP \\
\hline CDKN2B \\
\hline FAM160B2 \\
\hline TSPEAR \\
\hline RASGRF1 \\
\hline ELAVL1 \\
\hline HIST1H3E \\
\hline USP20 \\
\hline LEPROT \\
\hline MGRN1 \\
\hline HK2 \\
\hline GPR37L1 \\
\hline SLC16A8 \\
\hline CLCN2 \\
\hline LYRM7 \\
\hline GRAMD4 \\
\hline SEMA6C \\
\hline $\mathrm{C} 2 \mathrm{CD} 4 \mathrm{C}$ \\
\hline DDR1 \\
\hline
\end{tabular}

\begin{tabular}{|c|}
\hline NAIF1 \\
\hline ITGA11 \\
\hline AKNA \\
\hline SEC22C \\
\hline IRGQ \\
\hline CDC42EP1 \\
\hline HOXB3 \\
\hline GIPR \\
\hline CEMP1 \\
\hline RAB11FIP3 \\
\hline HOOK3 \\
\hline FAM222B \\
\hline OPRL1 \\
\hline TMEM109 \\
\hline COX6B2 \\
\hline SLC26A2 \\
\hline SGMS2 \\
\hline PIK3C2B \\
\hline CSPG4 \\
\hline ASIC4 \\
\hline TTLL12 \\
\hline ATF7IP \\
\hline MACC1 \\
\hline PPP1R12B \\
\hline GRK5 \\
\hline FOPNL \\
\hline TOX4 \\
\hline ZNF850 \\
\hline QPCTL \\
\hline NPAS1 \\
\hline DYNC1LI2 \\
\hline MIEF2 \\
\hline AVL9 \\
\hline ST5 \\
\hline SYT7 \\
\hline C4orf29 \\
\hline MITF \\
\hline FBXO45 \\
\hline UNC5A \\
\hline TBX20 \\
\hline C2orf71 \\
\hline TPCN1 \\
\hline FBXO31 \\
\hline KCNE4 \\
\hline NECAB3 \\
\hline C1RL \\
\hline KAT7 \\
\hline FAM212B \\
\hline ZRANB3 \\
\hline MYO1D \\
\hline ADAMTS13 \\
\hline SH3PXD2A \\
\hline RBBP4 \\
\hline TNFRSF25 \\
\hline NKPD1 \\
\hline STON2 \\
\hline IFNLR1 \\
\hline HEPACAM \\
\hline FAM43B \\
\hline UMODL1 \\
\hline CR1 \\
\hline C20orf144 \\
\hline
\end{tabular}

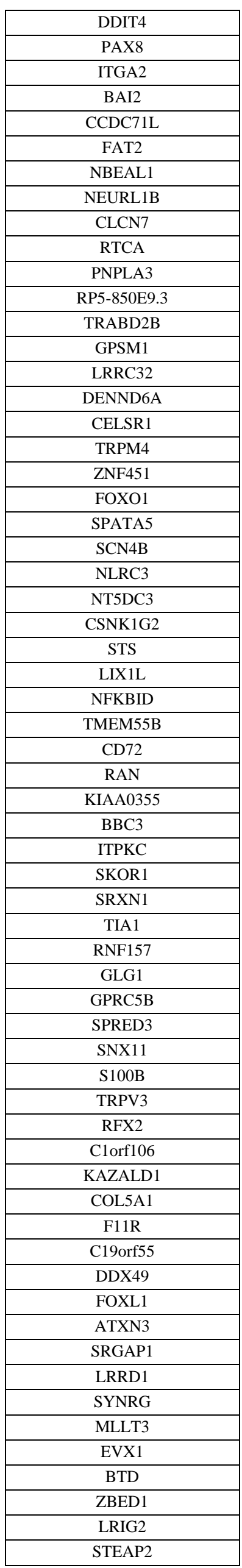

https://biointerfaceresearch.com/ 
https://doi.org/10.33263/BRIAC111.79437972

\begin{tabular}{|c|}
\hline ZFYVE20 \\
\hline RARA \\
\hline RUNX3 \\
\hline INTU \\
\hline ZNF816 \\
\hline GNPNAT1 \\
\hline TRUB2 \\
\hline KIAA0825 \\
\hline UNC13A \\
\hline NOL9 \\
\hline GSTK1 \\
\hline ZNF621 \\
\hline CENPI \\
\hline PGAM5 \\
\hline CACNA1A \\
\hline RNF114 \\
\hline EPS8L2 \\
\hline CD3EAP \\
\hline GRM6 \\
\hline STRA6 \\
\hline FRMD4A \\
\hline CPNE5 \\
\hline REEP3 \\
\hline SOX12 \\
\hline $\mathrm{CCDC} 127$ \\
\hline N4BP1 \\
\hline ACTR1A \\
\hline RAPGEF1 \\
\hline CARD8 \\
\hline ZNF710 \\
\hline UBOX5 \\
\hline NEUROG3 \\
\hline CEP135 \\
\hline ZNF629 \\
\hline ATAD5 \\
\hline ZMYM1 \\
\hline CEBPD \\
\hline SLC9A3R2 \\
\hline HAP1 \\
\hline ZNF460 \\
\hline COX19 \\
\hline C17orf51 \\
\hline ZNF207 \\
\hline MLLT1 \\
\hline REXO1 \\
\hline NTN1 \\
\hline RPUSD4 \\
\hline PDCL3 \\
\hline TRAF3IP2 \\
\hline RAD23B \\
\hline ZNF264 \\
\hline UHRF1BP1 \\
\hline GBX2 \\
\hline ITFG3 \\
\hline CACNA1I \\
\hline TMEM104 \\
\hline SLC7A1 \\
\hline CHMP1B \\
\hline PADI2 \\
\hline RPS6KA2 \\
\hline PACS1 \\
\hline NPY4R \\
\hline
\end{tabular}

\begin{tabular}{|c|}
\hline RNF7 \\
\hline CNNM3 \\
\hline$\overline{\mathrm{DSC}} 3$ \\
\hline SLC12A6 \\
\hline PEX13 \\
\hline PGPEP1 \\
\hline RUFY3 \\
\hline STK4 \\
\hline PHLDA3 \\
\hline SLC43A2 \\
\hline SRPX2 \\
\hline FAM163A \\
\hline TSPAN11 \\
\hline ZDBF2 \\
\hline NOP9 \\
\hline NDOR1 \\
\hline SPNS2 \\
\hline HK1 \\
\hline ELFN2 \\
\hline C2CD2L \\
\hline $\mathrm{TH}$ \\
\hline SH3GL1 \\
\hline PLEKHG4B \\
\hline ACSF3 \\
\hline SLC35A3 \\
\hline KIF26A \\
\hline ATCAY \\
\hline NARFL \\
\hline ATP6V1C2 \\
\hline PCDHB11 \\
\hline MLXIP \\
\hline PLCH2 \\
\hline PLA2G6 \\
\hline ELK4 \\
\hline KCNJ6 \\
\hline KCNAB2 \\
\hline KIAA1456 \\
\hline NKX6-2 \\
\hline SRM \\
\hline DPPA3 \\
\hline TONSL \\
\hline HSD17B13 \\
\hline MPRIP \\
\hline HIC2 \\
\hline AC021218.2 \\
\hline MYO9B \\
\hline AL049840.1 \\
\hline NXN \\
\hline PEX2 \\
\hline ELL \\
\hline ZNF155 \\
\hline RABL5 \\
\hline PLEKHA6 \\
\hline ZNF805 \\
\hline TMEM184A \\
\hline IKBIP \\
\hline DRAXIN \\
\hline CBX2 \\
\hline MTMR9 \\
\hline KIAA1210 \\
\hline TLN2 \\
\hline PTPN14 \\
\hline
\end{tabular}

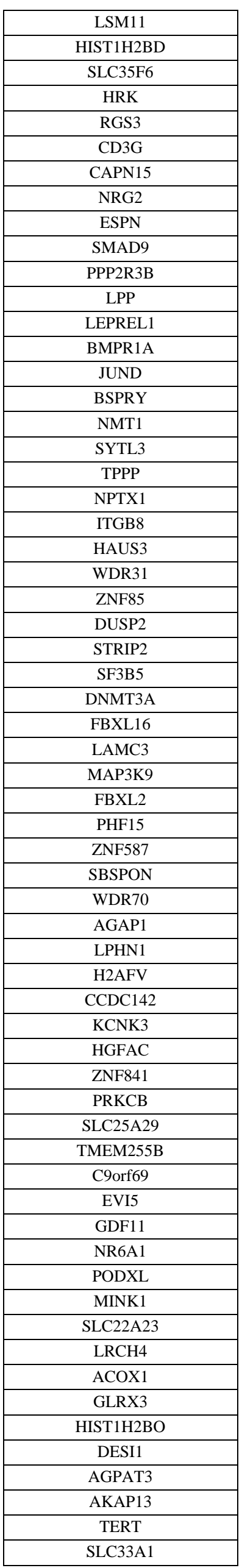

https://biointerfaceresearch.com/ 
https://doi.org/10.33263/BRIAC111.79437972

\begin{tabular}{|c|}
\hline LIMK1 \\
\hline PLEKHM2 \\
\hline PPARGC1B \\
\hline DNAJC3 \\
\hline XKR4 \\
\hline PLXNB1 \\
\hline TGFBR2 \\
\hline MIOX \\
\hline YY1 \\
\hline EXOSC2 \\
\hline ZNF730 \\
\hline PHAX \\
\hline ACAN \\
\hline DIP2C \\
\hline WNK2 \\
\hline EXOC8 \\
\hline GLYR1 \\
\hline RPP25 \\
\hline XIAP \\
\hline SDK1 \\
\hline DGKE \\
\hline ATG12 \\
\hline TMEM192 \\
\hline SMC5 \\
\hline CLN8 \\
\hline AGPAT6 \\
\hline HMX3 \\
\hline PDP2 \\
\hline ZNF442 \\
\hline KCTD20 \\
\hline KLHL11 \\
\hline DHODH \\
\hline SOX4 \\
\hline GREB1 \\
\hline LSM14A \\
\hline ZER1 \\
\hline MED9 \\
\hline FZD3 \\
\hline IGF2R \\
\hline RCAN3 \\
\hline RASSF6 \\
\hline TBRG1 \\
\hline UQCC1 \\
\hline SIT1 \\
\hline BCAR1 \\
\hline PAPOLG \\
\hline TFAP2B \\
\hline ATP5S \\
\hline ADAMTS17 \\
\hline CANT1 \\
\hline OSTM1 \\
\hline SFXN1 \\
\hline TEX22 \\
\hline OTUD7A \\
\hline GCLM \\
\hline SGPP2 \\
\hline AGAP2 \\
\hline MCM8 \\
\hline ANO7 \\
\hline VKORC1L1 \\
\hline ZNRF3 \\
\hline ZNF551 \\
\hline
\end{tabular}

\begin{tabular}{|c|}
\hline ISM2 \\
\hline AP5B1 \\
\hline LDLRAD2 \\
\hline RC3H1 \\
\hline SGSM3 \\
\hline RNASEH2C \\
\hline KCNQ4 \\
\hline MUC20 \\
\hline RASSF2 \\
\hline C3orf36 \\
\hline TMEM120B \\
\hline CSNK1A1 \\
\hline GXYLT2 \\
\hline SRD5A1 \\
\hline FAM131A \\
\hline GNA11 \\
\hline HMX2 \\
\hline AP1G1 \\
\hline ZNF738 \\
\hline IL17RD \\
\hline FOXE1 \\
\hline SNAP29 \\
\hline ANKRD33B \\
\hline MED13L \\
\hline ADAM19 \\
\hline KIF21B \\
\hline SAPCD2 \\
\hline SERINC3 \\
\hline QSOX1 \\
\hline FBXO48 \\
\hline FGD4 \\
\hline SIRT5 \\
\hline ARHGEF15 \\
\hline FBXO10 \\
\hline VENTX \\
\hline VWA5B1 \\
\hline HSPG2 \\
\hline DGCR2 \\
\hline MYLK3 \\
\hline ZNF585B \\
\hline DGKD \\
\hline LRCH3 \\
\hline ZNF696 \\
\hline PTPRS \\
\hline COL8A2 \\
\hline HECTD3 \\
\hline ATP5G1 \\
\hline NDUFA7 \\
\hline IKZF3 \\
\hline SLC12A7 \\
\hline DYM \\
\hline SDF4 \\
\hline RAD1 \\
\hline IMPAD1 \\
\hline TRAPPC2P1 \\
\hline BNIP2 \\
\hline MANEAL \\
\hline GRM1 \\
\hline THSD4 \\
\hline WDR12 \\
\hline TNFRSF14 \\
\hline ABHD15 \\
\hline
\end{tabular}

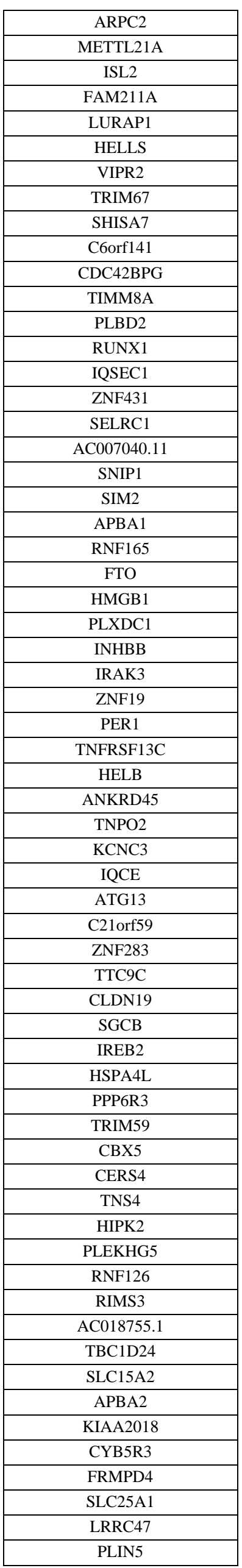

https://biointerfaceresearch.com/ 
https://doi.org/10.33263/BRIAC111.79437972

\begin{tabular}{|c|}
\hline GLDN \\
\hline CPLX1 \\
\hline AGTRAP \\
\hline EPHA10 \\
\hline ZC3H8 \\
\hline NOVA2 \\
\hline LONRF2 \\
\hline TMX4 \\
\hline TBC1D16 \\
\hline ZBTB20 \\
\hline PHACTR4 \\
\hline PHACTR1 \\
\hline PANK4 \\
\hline ZDHHC3 \\
\hline ZNF799 \\
\hline PPM1K \\
\hline AP5S1 \\
\hline C4orf33 \\
\hline ONECUT3 \\
\hline HOXD11 \\
\hline ZBTB3 \\
\hline EXPH5 \\
\hline SH3BP2 \\
\hline HDLBP \\
\hline COL9A2 \\
\hline ZDHHC24 \\
\hline PPP1R16B \\
\hline AAK1 \\
\hline PRICKLE1 \\
\hline CENPN \\
\hline NONO \\
\hline RPL18A \\
\hline EHBP1L1 \\
\hline GNB4 \\
\hline CAMK4 \\
\hline BCORL1 \\
\hline HSPA5 \\
\hline FBXL20 \\
\hline DCP2 \\
\hline TRPM6 \\
\hline MOB4 \\
\hline SLC9A7 \\
\hline LMTK2 \\
\hline GDF7 \\
\hline SZT2 \\
\hline AMDHD2 \\
\hline ZNF546 \\
\hline P2RY2 \\
\hline DARS2 \\
\hline ENOSF1 \\
\hline FCF1 \\
\hline MAGI1 \\
\hline SPIRE2 \\
\hline METTL16 \\
\hline GK5 \\
\hline LRRK1 \\
\hline GAS7 \\
\hline UTP6 \\
\hline EIF3L \\
\hline ZNF35 \\
\hline BPTF \\
\hline C12orf5 \\
\hline
\end{tabular}

\begin{tabular}{|c|}
\hline PVR \\
\hline MYH9 \\
\hline KLF16 \\
\hline SP6 \\
\hline CMKLR1 \\
\hline CENPP \\
\hline NOL10 \\
\hline IGF1R \\
\hline HIST3H2BB \\
\hline GCNT4 \\
\hline HS3ST1 \\
\hline FAM83F \\
\hline CAPZB \\
\hline C17orf75 \\
\hline PAFAH1B1 \\
\hline RANBP3 \\
\hline FASTKD2 \\
\hline MNT \\
\hline MMS22L \\
\hline ARSG \\
\hline CLCN5 \\
\hline MPPE1 \\
\hline PRSS21 \\
\hline MAGI3 \\
\hline PDK3 \\
\hline C19orf52 \\
\hline KLHDC10 \\
\hline COLGALT2 \\
\hline CAMSAP1 \\
\hline C15orf38 \\
\hline RWDD1 \\
\hline GMPPB \\
\hline THAP1 \\
\hline TRAF6 \\
\hline RXRA \\
\hline RPTOR \\
\hline RAB10 \\
\hline NUP205 \\
\hline UGGT1 \\
\hline USH1G \\
\hline CLMN \\
\hline TSHZ2 \\
\hline EFR3B \\
\hline UTP15 \\
\hline COPA \\
\hline MTUS2 \\
\hline NEUROD2 \\
\hline HSD17B12 \\
\hline KLHL24 \\
\hline KCND3 \\
\hline PPIL6 \\
\hline RAB11FIP4 \\
\hline ZFP30 \\
\hline RBM28 \\
\hline TNRC6B \\
\hline POU2F1 \\
\hline DCPS \\
\hline SEMA3E \\
\hline TRIP11 \\
\hline VPS13B \\
\hline NTNG2 \\
\hline GPR153 \\
\hline
\end{tabular}

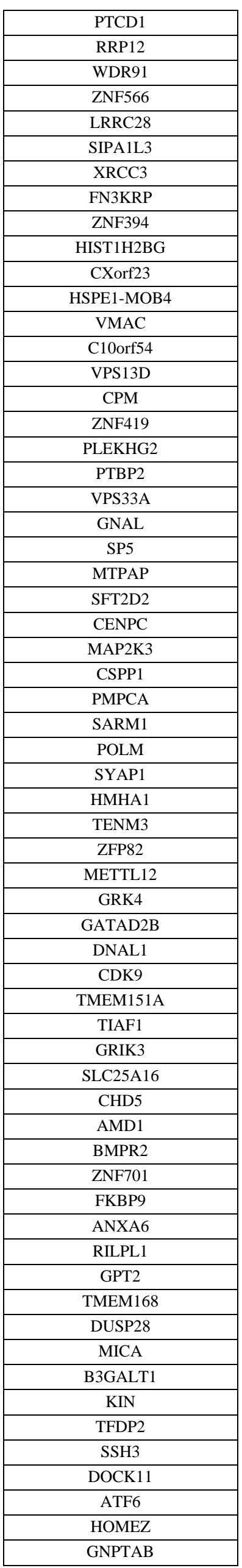

https://biointerfaceresearch.com/ 
https://doi.org/10.33263/BRIAC111.79437972

\begin{tabular}{|c|}
\hline SMS \\
\hline EHD2 \\
\hline SIAE \\
\hline SLC4A8 \\
\hline ZNF347 \\
\hline AGO3 \\
\hline SLC25A36 \\
\hline ZSCAN29 \\
\hline SLC2A5 \\
\hline PIGQ \\
\hline HHIP \\
\hline POTEM \\
\hline POTEG \\
\hline BRCC3 \\
\hline NEDD4L \\
\hline FAM162A \\
\hline YIPF2 \\
\hline NCAM1 \\
\hline LHFPL4 \\
\hline ZNF446 \\
\hline TANC2 \\
\hline SLC7A11 \\
\hline PLXNA1 \\
\hline NPR1 \\
\hline LPAR3 \\
\hline C15orf40 \\
\hline TOPBP1 \\
\hline SDHAF1 \\
\hline ZNF708 \\
\hline ZSWIM7 \\
\hline CCDC93 \\
\hline ANAPC16 \\
\hline OGFOD3 \\
\hline CIAO1 \\
\hline MEX3A \\
\hline MYO18A \\
\hline LHX1 \\
\hline ARHGAP27 \\
\hline CLUAP1 \\
\hline MAP1LC3B \\
\hline SUMO2 \\
\hline TCEANC2 \\
\hline SMAD4 \\
\hline ABHD2 \\
\hline MKI67IP \\
\hline RNF41 \\
\hline GNG7 \\
\hline GDAP1L1 \\
\hline TMEM220 \\
\hline TMEM186 \\
\hline INPP5K \\
\hline KLHL4 \\
\hline TTC4 \\
\hline NTRK3 \\
\hline NFRKB \\
\hline GTF3C6 \\
\hline USP13 \\
\hline GEMIN6 \\
\hline FASN \\
\hline SNRPD1 \\
\hline CLSTN2 \\
\hline CLPB \\
\hline
\end{tabular}

\begin{tabular}{|c|}
\hline BDH1 \\
\hline SUSD1 \\
\hline TPCN2 \\
\hline ZSCAN2 \\
\hline SF3B1 \\
\hline MYCBP \\
\hline KIAA1551 \\
\hline WBSCR27 \\
\hline CELSR2 \\
\hline ZNF766 \\
\hline SWSAP1 \\
\hline GNAI3 \\
\hline ZNF324B \\
\hline PLXNA3 \\
\hline IBA57 \\
\hline HABP2 \\
\hline URM1 \\
\hline NETO2 \\
\hline LETM1 \\
\hline TBRG4 \\
\hline SNCA \\
\hline RBBP8 \\
\hline SERPINA4 \\
\hline FKRP \\
\hline POLR1C \\
\hline ADH5 \\
\hline B4GALT7 \\
\hline RSBN1L \\
\hline TAOK1 \\
\hline PDE12 \\
\hline GABRB3 \\
\hline PHC3 \\
\hline C19orf47 \\
\hline MTMR10 \\
\hline PURB \\
\hline ZHX3 \\
\hline GGCX \\
\hline ZNF790 \\
\hline TIMM50 \\
\hline NFE2L1 \\
\hline IL18BP \\
\hline LANCL3 \\
\hline CD248 \\
\hline SHB \\
\hline E2F3 \\
\hline ARHGAP35 \\
\hline EPHA8 \\
\hline ANKRD13B \\
\hline ANK1 \\
\hline FLJ27365 \\
\hline ERN1 \\
\hline ALPK3 \\
\hline NAV2 \\
\hline C20orf194 \\
\hline GALNT10 \\
\hline GTF2F1 \\
\hline PSKH1 \\
\hline RPL14 \\
\hline CC2D1B \\
\hline LIG3 \\
\hline TSPAN14 \\
\hline ANGEL2 \\
\hline
\end{tabular}

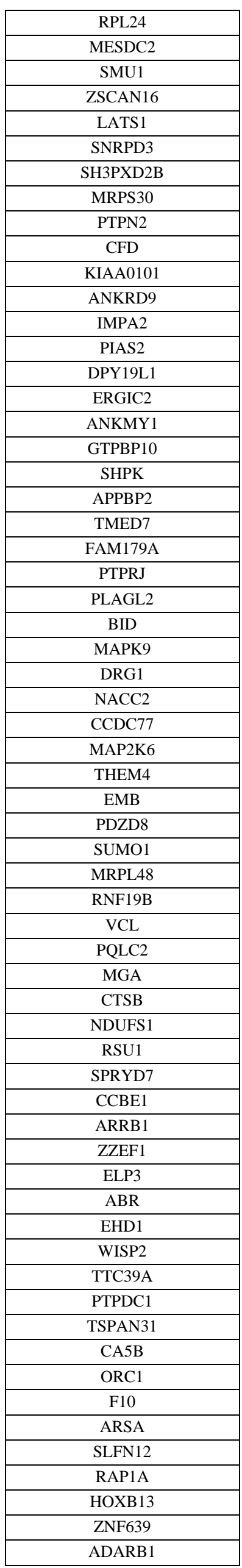

https://biointerfaceresearch.com/ 
https://doi.org/10.33263/BRIAC111.79437972

\begin{tabular}{|c|}
\hline TSKU \\
\hline ADHFE1 \\
\hline MYPN \\
\hline GADD45GIP1 \\
\hline IRF1 \\
\hline ALDH6A1 \\
\hline NDUFC1 \\
\hline ELP6 \\
\hline SOCS7 \\
\hline LAYN \\
\hline ST3GAL6 \\
\hline CEP68 \\
\hline TLE3 \\
\hline PTCHD1 \\
\hline SOD2 \\
\hline FANCA \\
\hline CDK4 \\
\hline LIMS1 \\
\hline SLC16A10 \\
\hline SCIN \\
\hline C7orf55-LUC7L2 \\
\hline RAB13 \\
\hline SLC1A5 \\
\hline MGP \\
\hline HJURP \\
\hline CDH6 \\
\hline ATP5F1 \\
\hline C17orf85 \\
\hline HLX \\
\hline MON2 \\
\hline AGTPBP1 \\
\hline ADRBK2 \\
\hline RBM4B \\
\hline ABCF1 \\
\hline DDX19B \\
\hline SIRT3 \\
\hline SARDH \\
\hline EMC7 \\
\hline SBNO1 \\
\hline CNKSR3 \\
\hline DNAJC21 \\
\hline C1orf21 \\
\hline SDCCAG3 \\
\hline SORCS2 \\
\hline SLC35F5 \\
\hline NUP43 \\
\hline NAGS \\
\hline NXPE3 \\
\hline ABHD6 \\
\hline BZW1 \\
\hline PRIM1 \\
\hline $\mathrm{AQR}$ \\
\hline IMP4 \\
\hline ETFDH \\
\hline LUC7L2 \\
\hline XPNPEP3 \\
\hline AK1 \\
\hline ZNF445 \\
\hline TXNL4A \\
\hline SLC38A9 \\
\hline GPR133 \\
\hline PDE7A \\
\hline
\end{tabular}

\begin{tabular}{|c|}
\hline FOXRED2 \\
\hline TGS1 \\
\hline SOX11 \\
\hline CHSY3 \\
\hline GSR \\
\hline NDUFA4 \\
\hline POLR3B \\
\hline CCSER2 \\
\hline NADSYN1 \\
\hline MED28 \\
\hline NABP1 \\
\hline ZNF259 \\
\hline PCM1 \\
\hline GTPBP4 \\
\hline LPAR2 \\
\hline RPP14 \\
\hline PRR24 \\
\hline VPS18 \\
\hline RP1-170O19.20 \\
\hline TTF2 \\
\hline SMAD2 \\
\hline FAM83D \\
\hline HOXA9 \\
\hline F2RL1 \\
\hline APOA1 \\
\hline SIK2 \\
\hline ODF2L \\
\hline PPM1D \\
\hline NME1-NME2 \\
\hline FLYWCH2 \\
\hline C5orf55 \\
\hline HSDL2 \\
\hline CENPM \\
\hline AGXT2 \\
\hline ABCG8 \\
\hline MAML2 \\
\hline MRPS14 \\
\hline CLYBL \\
\hline GTDC1 \\
\hline FANCM \\
\hline ATG2B \\
\hline C14orf80 \\
\hline NME2 \\
\hline ZNF177 \\
\hline TXNL1 \\
\hline P4HA2 \\
\hline PSD4 \\
\hline ABCG2 \\
\hline USP1 \\
\hline APOPT1 \\
\hline CTSV \\
\hline ATP6V0E1 \\
\hline FGFR1OP \\
\hline PYCR1 \\
\hline NNMT \\
\hline CALCOCO2 \\
\hline MTHFR \\
\hline PLAC8 \\
\hline MPI \\
\hline SLC35C2 \\
\hline PHB2 \\
\hline NDUFV3 \\
\hline
\end{tabular}

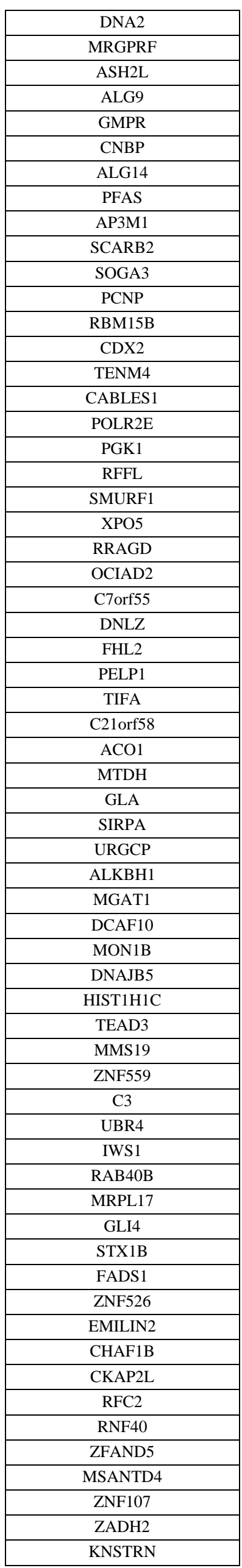

https://biointerfaceresearch.com/ 
https://doi.org/10.33263/BRIAC111.79437972

\begin{tabular}{|c|}
\hline VGLL3 \\
\hline SRSF9 \\
\hline TBC1D15 \\
\hline NSUN3 \\
\hline IPO9 \\
\hline PTGR2 \\
\hline TIGD2 \\
\hline COMTD1 \\
\hline COPS3 \\
\hline CKAP4 \\
\hline CEP63 \\
\hline LMF1 \\
\hline RMDN1 \\
\hline SELK \\
\hline AIG1 \\
\hline FYTTD1 \\
\hline CHSY1 \\
\hline SSBP2 \\
\hline YME1L1 \\
\hline RRM2 \\
\hline KB-1507C5.2 \\
\hline INO80 \\
\hline SNAPC3 \\
\hline HINFP \\
\hline RAD51L3-RFFL \\
\hline RBFA \\
\hline ZNF668 \\
\hline HMGB2 \\
\hline SLC8A1 \\
\hline HAS2 \\
\hline DSN1 \\
\hline TOP3A \\
\hline SHOC2 \\
\hline C16orf72 \\
\hline TTC38 \\
\hline PAN2 \\
\hline RNF24 \\
\hline C4orf32 \\
\hline SGOL1 \\
\hline NTMT1 \\
\hline ECHDC3 \\
\hline PRPF6 \\
\hline RELT \\
\hline CMBL \\
\hline FBXO3 \\
\hline TMEM86B \\
\hline SKP1 \\
\hline ZNF607 \\
\hline CADM1 \\
\hline SERPING1 \\
\hline LYRM4 \\
\hline MTO1 \\
\hline TRIOBP \\
\hline EMC2 \\
\hline NCKAP1 \\
\hline SLBP \\
\hline DNAJC30 \\
\hline ENAH \\
\hline SLC30A5 \\
\hline DDI2 \\
\hline NUPL2 \\
\hline SCO1 \\
\hline
\end{tabular}

\begin{tabular}{|c|}
\hline PCDHB2 \\
\hline GPRIN3 \\
\hline TMEM135 \\
\hline HTT \\
\hline CORO7 \\
\hline PPIL2 \\
\hline DOCK7 \\
\hline TM4SF5 \\
\hline RASSF4 \\
\hline DR1 \\
\hline KCMF1 \\
\hline ITGA1 \\
\hline MAPK8IP2 \\
\hline MRPS23 \\
\hline ANKLE2 \\
\hline GLP2R \\
\hline ARFIP2 \\
\hline EIF4E \\
\hline CYB5D1 \\
\hline TTC13 \\
\hline TMEM134 \\
\hline ACTR10 \\
\hline ATP6V1E1 \\
\hline LMAN2L \\
\hline MED16 \\
\hline PRCD \\
\hline DBNL \\
\hline METTL22 \\
\hline MTHFD1 \\
\hline NINJ1 \\
\hline CYB5R4 \\
\hline AGT \\
\hline TFCP2 \\
\hline MMP15 \\
\hline CAND1 \\
\hline MAPK1 \\
\hline ZMYM4 \\
\hline DNAJC10 \\
\hline CDS2 \\
\hline RPL37A \\
\hline PRPS1 \\
\hline FNDC3B \\
\hline AC106017.1 \\
\hline NEK9 \\
\hline TMEM106B \\
\hline RNF115 \\
\hline G3BP2 \\
\hline POLA2 \\
\hline GRWD1 \\
\hline DCUN1D5 \\
\hline DYRK1B \\
\hline RNMTL1 \\
\hline SERPINH1 \\
\hline TNPO1 \\
\hline WDR88 \\
\hline LDHA \\
\hline TIMM10 \\
\hline CAPZA2 \\
\hline ORMDL2 \\
\hline DNTTIP2 \\
\hline NLN \\
\hline CHD7 \\
\hline
\end{tabular}

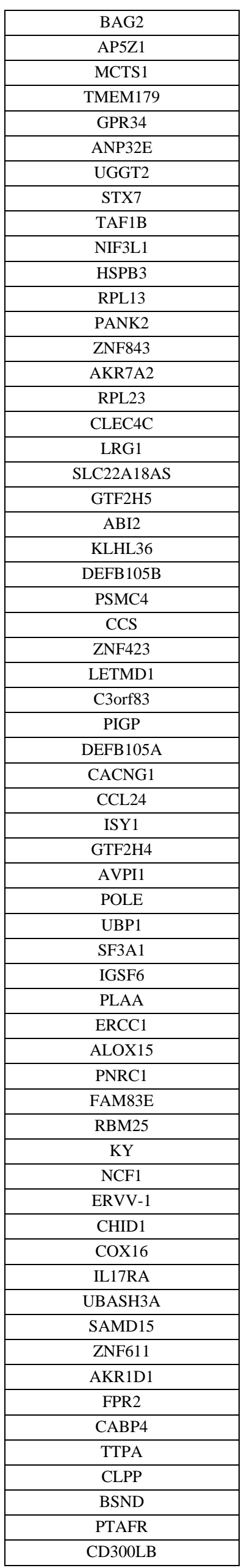

https://biointerfaceresearch.com/ 
https://doi.org/10.33263/BRIAC111.79437972

\begin{tabular}{|c|}
\hline IL12RB2 \\
\hline MYOZ2 \\
\hline LUC7L3 \\
\hline CDNF \\
\hline RBM23 \\
\hline LHPP \\
\hline HIF1A \\
\hline CD84 \\
\hline CD226 \\
\hline C10orf71 \\
\hline FPR1 \\
\hline FHL5 \\
\hline PRPF4 \\
\hline MBD5 \\
\hline ACOT2 \\
\hline TLK2 \\
\hline AGAP9 \\
\hline GABRR2 \\
\hline GRAP2 \\
\hline CRIPT \\
\hline DDX4 \\
\hline PPIL4 \\
\hline AKAP1 \\
\hline RANBP3L \\
\hline NCL \\
\hline MYBPC1 \\
\hline ATIC \\
\hline TRA2B \\
\hline ABCB11 \\
\hline GLTSCR2 \\
\hline SLFN12L \\
\hline EPHX2 \\
\hline FDFT1 \\
\hline GPR78 \\
\hline SPATS2L \\
\hline BTLA \\
\hline SLC22A12 \\
\hline YIF1B \\
\hline GRM4 \\
\hline MAMDC4 \\
\hline SND1 \\
\hline KLHDC8A \\
\hline ABCA6 \\
\hline TIMM23 \\
\hline SLC24A4 \\
\hline CRP \\
\hline
\end{tabular}

\begin{tabular}{|c|}
\hline TG \\
\hline EMG1 \\
\hline NLRP9 \\
\hline ASB6 \\
\hline AC135178.1 \\
\hline TIMD4 \\
\hline SRSF3 \\
\hline CD3D \\
\hline NDUFB5 \\
\hline C9orf3 \\
\hline MTMR8 \\
\hline ZNF417 \\
\hline SHC3 \\
\hline MYO1F \\
\hline SEMA4F \\
\hline RBP4 \\
\hline TRIM72 \\
\hline SLC16A3 \\
\hline BCDIN3D \\
\hline ATP5J2-PTCD1 \\
\hline EMC1 \\
\hline SMTNL2 \\
\hline SPTLC3 \\
\hline PARP2 \\
\hline ZFP36L2 \\
\hline SLC22A15 \\
\hline RCN2 \\
\hline SSTR2 \\
\hline UQCR11 \\
\hline GPI \\
\hline ZNF8 \\
\hline SELPLG \\
\hline NRXN3 \\
\hline ALOX5AP \\
\hline ALDOA \\
\hline $\mathrm{UBC}$ \\
\hline C1orf210 \\
\hline BRIP1 \\
\hline PRMT7 \\
\hline TFB1M \\
\hline MTFMT \\
\hline PRR11 \\
\hline PSMB9 \\
\hline DNAH17 \\
\hline C18orf32 \\
\hline CPNE2 \\
\hline
\end{tabular}

\begin{tabular}{|c|}
\hline $\mathrm{CCNO}$ \\
\hline VASN \\
\hline KLF2 \\
\hline CAPRIN1 \\
\hline RLIM \\
\hline CYP20A1 \\
\hline NKD2 \\
\hline NAA50 \\
\hline ANKFY1 \\
\hline FOSL2 \\
\hline TRIM38 \\
\hline SRSF1 \\
\hline NQO2 \\
\hline S100A16 \\
\hline MXRA8 \\
\hline PPAP2B \\
\hline CCNT1 \\
\hline NUP93 \\
\hline KIF1C \\
\hline MSRB2 \\
\hline ATP5G3 \\
\hline ST20-MTHFS \\
\hline STRADA \\
\hline ZNF326 \\
\hline PEX16 \\
\hline POLD4 \\
\hline WDR13 \\
\hline HEYL \\
\hline C1QTNF8 \\
\hline ZNF426 \\
\hline PLA2G16 \\
\hline RIMKLA \\
\hline PPDPF \\
\hline 6-Mar \\
\hline MTHFS \\
\hline SC5D \\
\hline USP4 \\
\hline HSPA1B \\
\hline VCAM1 \\
\hline MRPL15 \\
\hline XBP1 \\
\hline KIAA0408 \\
\hline ARRDC2 \\
\hline GM2A \\
\hline OSTF1 \\
\hline
\end{tabular}


SupplyTable 2

\begin{tabular}{|c|c|c|c|c|c|}
\hline \multicolumn{4}{|l|}{ \#\#Databases: KEGG PATHWAY } & & \\
\hline \multicolumn{4}{|c|}{ \#\#Statistical test method: hypergeometric test / Fisher's exact test } & & \\
\hline \multicolumn{6}{|c|}{ \#\#FDR correction method: Benjamini and Hochberg } \\
\hline \#Term & ID & $\begin{array}{c}\text { Input } \\
\text { number }\end{array}$ & $\begin{array}{c}\text { Background } \\
\text { number }\end{array}$ & P-Value & $\begin{array}{l}\text { Corrected P- } \\
\text { Value } \\
\end{array}$ \\
\hline Pathways in cancer & hsa05200 & 50 & 397 & $1.25 \mathrm{E}-09$ & $2.33 \mathrm{E}-08$ \\
\hline Rap1 signaling pathway & hsa04015 & 32 & 211 & $2.64 \mathrm{E}-08$ & 4.24E-07 \\
\hline PI3K-Akt signaling pathway & hsa04151 & 41 & 342 & $1.25 \mathrm{E}-07$ & $1.85 \mathrm{E}-06$ \\
\hline Ras signaling pathway & hsa04014 & 31 & 228 & $3.78 \mathrm{E}-07$ & $5.17 \mathrm{E}-06$ \\
\hline Transcriptional misregulation in cancer & hsa05202 & 27 & 180 & $3.8 \mathrm{E}-07$ & 5.19E-06 \\
\hline Neuroactive ligand-receptor interaction & hsa04080 & 34 & 278 & $9.6 \mathrm{E}-07$ & $1.21 \mathrm{E}-05$ \\
\hline MAPK signaling pathway & hsa04010 & 32 & 255 & $1.2 \mathrm{E}-06$ & $1.49 \mathrm{E}-05$ \\
\hline $\begin{array}{l}\text { Signaling pathways regulating } \\
\text { pluripotency of stem cells }\end{array}$ & hsa04550 & 22 & 142 & $2.76 \mathrm{E}-06$ & $3.18 \mathrm{E}-05$ \\
\hline Regulation of actin cytoskeleton & hsa04810 & 28 & 215 & $2.87 \mathrm{E}-06$ & $3.29 \mathrm{E}-05$ \\
\hline Metabolic pathways & hsa01100 & 94 & 1243 & $3.62 \mathrm{E}-06$ & $4.06 \mathrm{E}-05$ \\
\hline TNF signaling pathway & hsa04668 & 17 & 110 & $3.71 \mathrm{E}-05$ & 0.000338 \\
\hline FoxO signaling pathway & hsa04068 & 19 & 134 & $3.86 \mathrm{E}-05$ & 0.000348 \\
\hline Renal cell carcinoma & hsa05211 & 13 & 67 & $3.9 \mathrm{E}-05$ & 0.000351 \\
\hline Glycine serine and threonine metabolism & hsa00260 & 10 & 40 & $4.87 \mathrm{E}-05$ & 0.000432 \\
\hline Influenza A & hsa05164 & 22 & 176 & $5.56 \mathrm{E}-05$ & 0.000485 \\
\hline $\begin{array}{l}\text { Longevity regulating pathway - multiple } \\
\text { species }\end{array}$ & hsa04213 & 12 & 64 & 0.000102 & 0.000834 \\
\hline Insulin resistance & hsa04931 & 16 & 109 & 0.000107 & 0.000868 \\
\hline Measles & hsa05162 & 18 & 136 & 0.000134 & 0.001052 \\
\hline Apoptosis & hsa04210 & 18 & 140 & 0.000186 & 0.001392 \\
\hline p53 signaling pathway & hsa04115 & 12 & 69 & 0.000191 & 0.00142 \\
\hline mTOR signaling pathway & hsa04150 & 19 & 154 & 0.000202 & 0.001497 \\
\hline Circadian rhythm & hsa04710 & 8 & 31 & 0.00023 & 0.001674 \\
\hline beta-Alanine metabolism & hsa00410 & 8 & 31 & 0.00023 & 0.001674 \\
\hline Longevity regulating pathway & hsa04211 & 14 & 94 & 0.000247 & 0.001783 \\
\hline Jak-STAT signaling pathway & hsa04630 & 19 & 158 & 0.000271 & 0.001926 \\
\hline Endocytosis & hsa04144 & 26 & 260 & 0.000344 & 0.002355 \\
\hline Axon guidance & hsa04360 & 20 & 176 & 0.000371 & 0.00251 \\
\hline Alcoholism & hsa05034 & 20 & 179 & 0.000452 & 0.002974 \\
\hline Hippo signaling pathway & hsa04390 & 18 & 154 & 0.000527 & 0.003419 \\
\hline HTLV-I infection & hsa05166 & 25 & 259 & 0.000709 & 0.004375 \\
\hline Notch signaling pathway & hsa04330 & 9 & 48 & 0.000736 & 0.004529 \\
\hline Hepatitis C & hsa05160 & 16 & 133 & 0.000794 & 0.004799 \\
\hline Focal adhesion & hsa04510 & 21 & 203 & 0.000824 & 0.004956 \\
\hline Inositol phosphate metabolism & hsa00562 & 11 & 71 & 0.000827 & 0.004962 \\
\hline Prolactin signaling pathway & hsa04917 & 11 & 72 & 0.000916 & 0.005352 \\
\hline Arginine and proline metabolism & hsa00330 & 9 & 50 & 0.000951 & 0.005513 \\
\hline Estrogen signaling pathway & hsa04915 & 13 & 99 & 0.001167 & 0.006583 \\
\hline Melanogenesis & hsa04916 & 13 & 100 & 0.001267 & 0.00707 \\
\hline cAMP signaling pathway & hsa04024 & 20 & 199 & 0.001488 & 0.008019 \\
\hline Protein digestion and absorption & hsa04974 & 12 & 90 & 0.001581 & 0.008429 \\
\hline Basal cell carcinoma & hsa05217 & 9 & 55 & 0.001718 & 0.008992 \\
\hline EGFR tyrosine kinase inhibitor resistance & hsa01521 & 11 & 81 & 0.002141 & 0.010727 \\
\hline Selenocompound metabolism & hsa00450 & 5 & 17 & 0.002178 & 0.010861 \\
\hline Melanoma & hsa05218 & 10 & 71 & 0.002642 & 0.012742 \\
\hline TGF-beta signaling pathway & hsa04350 & 11 & 84 & 0.002765 & 0.013183 \\
\hline Endocrine resistance & hsa01522 & 12 & 97 & 0.002782 & 0.01325 \\
\hline Dorso-ventral axis formation & hsa04320 & 6 & 28 & 0.003142 & 0.014698 \\
\hline $\begin{array}{l}\text { Aldosterone-regulated sodium } \\
\text { reabsorption }\end{array}$ & hsa04960 & 7 & 39 & 0.003489 & 0.015961 \\
\hline Herpes simplex infection & hsa05168 & 18 & 186 & 0.003607 & 0.016437 \\
\hline Chemokine signaling pathway & hsa04062 & 18 & 187 & 0.003798 & 0.017195 \\
\hline Hematopoietic cell lineage & hsa04640 & 11 & 88 & 0.003817 & 0.017263 \\
\hline Tryptophan metabolism & hsa00380 & 7 & 40 & 0.003948 & 0.017598 \\
\hline Prostate cancer & $\mathrm{hsa} 05215$ & 11 & 89 & 0.004124 & 0.018265 \\
\hline Lysine degradation & hsa00310 & 8 & 52 & 0.004273 & 0.018772 \\
\hline HIF-1 signaling pathway & hsa04066 & 12 & 103 & 0.004322 & 0.018943 \\
\hline Thyroid hormone signaling pathway & hsa04919 & 13 & 118 & 0.004692 & 0.02039 \\
\hline Toxoplasmosis & hsa05145 & 13 & 119 & 0.005003 & 0.021516 \\
\hline Legionellosis & hsa05134 & 8 & 55 & 0.005771 & 0.023912 \\
\hline ECM-receptor interaction & hsa04512 & 10 & 82 & 0.006618 & 0.026847 \\
\hline ABC transporters & hsa02010 & 7 & 45 & 0.006965 & 0.027929 \\
\hline Cysteine and methionine metabolism & $\mathrm{hsa00270}$ & 7 & 45 & 0.006965 & 0.027929 \\
\hline Acute myeloid leukemia & hsa05221 & 8 & 57 & 0.006972 & 0.027929 \\
\hline Peroxisome & hsa04146 & 10 & 83 & 0.007135 & 0.028504 \\
\hline Adipocytokine signaling pathway & hsa04920 & 9 & 70 & 0.00721 & 0.028693 \\
\hline
\end{tabular}


https://doi.org/10.33263/BRIAC111.79437972

\begin{tabular}{|c|c|c|c|c|c|}
\hline Cytokine-cytokine receptor interaction & hsa04060 & 22 & 265 & 0.007523 & 0.029798 \\
\hline $\begin{array}{l}\text { Inflammatory mediator regulation of TRP } \\
\text { channels }\end{array}$ & hsa04750 & 11 & 98 & 0.007864 & 0.030617 \\
\hline $\begin{array}{c}\text { Alanine aspartate and glutamate } \\
\text { metabolism }\end{array}$ & hsa00250 & 6 & 35 & 0.008121 & 0.031442 \\
\hline $\begin{array}{c}\text { Glycosphingolipid biosynthesis - lacto and } \\
\text { neolacto series }\end{array}$ & hsa00601 & 5 & 26 & 0.010174 & 0.037761 \\
\hline Natural killer cell mediated cytotoxicity & hsa04650 & 13 & 135 & 0.012616 & 0.044682 \\
\hline Sphingolipid signaling pathway & hsa04071 & 12 & 121 & 0.01328 & 0.046632 \\
\hline MicroRNAs in cancer & hsa05206 & 23 & 299 & 0.014109 & 0.048345 \\
\hline Insulin signaling pathway & hsa04910 & 13 & 139 & 0.015478 & 0.052197 \\
\hline Aldosterone synthesis and secretion & hsa04925 & 9 & 81 & 0.016259 & 0.054134 \\
\hline AMPK signaling pathway & hsa04152 & 12 & 125 & 0.016465 & 0.054683 \\
\hline RNA transport & hsa03013 & 15 & 172 & 0.016831 & 0.055118 \\
\hline Wnt signaling pathway & hsa04310 & 13 & 143 & 0.018809 & 0.060254 \\
\hline Glutamatergic synapse & hsa04724 & 11 & 114 & 0.020436 & 0.06469 \\
\hline Huntington's disease & hsa05016 & 16 & 193 & 0.020656 & 0.06469 \\
\hline Insulin secretion & hsa04911 & 9 & 85 & 0.021036 & 0.065383 \\
\hline Amoebiasis & hsa05146 & 10 & 100 & 0.021533 & 0.066595 \\
\hline $\begin{array}{l}\text { AGE-RAGE signaling pathway in diabetic } \\
\text { complications }\end{array}$ & hsa04933 & 10 & 101 & 0.022777 & 0.069597 \\
\hline Calcium signaling pathway & hsa04020 & 15 & 180 & 0.023569 & 0.071684 \\
\hline Glucagon signaling pathway & hsa04922 & 10 & 102 & 0.024071 & 0.073043 \\
\hline cGMP-PKG signaling pathway & hsa04022 & 14 & 167 & 0.026908 & 0.079495 \\
\hline Cocaine addiction & hsa05030 & 6 & 49 & 0.031476 & 0.089796 \\
\hline Synaptic vesicle cycle & hsa04721 & 7 & 63 & 0.032031 & 0.090826 \\
\hline Viral carcinogenesis & hsa05203 & 16 & 205 & 0.032441 & 0.091904 \\
\hline Proteoglycans in cancer & hsa05205 & 16 & 205 & 0.032441 & 0.091904 \\
\hline Ovarian steroidogenesis & hsa04913 & 6 & 50 & 0.034005 & 0.094755 \\
\hline Caffeine metabolism & hsa00232 & 2 & 5 & 0.034217 & 0.094755 \\
\hline Glioma & hsa05214 & 7 & 65 & 0.036576 & 0.098824 \\
\hline Aminoacyl-tRNA biosynthesis & hsa00970 & 7 & 66 & 0.038999 & 0.104571 \\
\hline Phospholipase D signaling pathway & hsa04072 & 12 & 144 & 0.039855 & 0.105692 \\
\hline $\begin{array}{l}\text { Glycosaminoglycan biosynthesis - keratan } \\
\text { sulfate }\end{array}$ & hsa00533 & 3 & 15 & 0.040964 & 0.107372 \\
\hline Amphetamine addiction & hsa05031 & 7 & 67 & 0.041525 & 0.108429 \\
\hline Hepatitis B & hsa05161 & 12 & 146 & 0.043236 & 0.111925 \\
\hline Vitamin B6 metabolism & hsa00750 & 2 & 6 & 0.044328 & 0.111925 \\
\hline Osteoclast differentiation & hsa04380 & 11 & 132 & 0.047675 & 0.118419 \\
\hline $\begin{array}{c}\text { Protein processing in endoplasmic } \\
\text { reticulum }\end{array}$ & hsa04141 & 13 & 166 & 0.049148 & 0.121063 \\
\hline Regulation of lipolysis in adipocytes & hsa04923 & 6 & 56 & 0.051909 & 0.127111 \\
\hline Non-small cell lung cancer & hsa05223 & 6 & 56 & 0.051909 & 0.127111 \\
\hline Leukocyte transendothelial migration & hsa04670 & 10 & 118 & 0.052511 & 0.128347 \\
\hline Epstein-Barr virus infection & hsa05169 & 15 & 204 & 0.055782 & 0.130732 \\
\hline Systemic lupus erythematosus & hsa05322 & 11 & 136 & 0.056053 & 0.131301 \\
\hline Neurotrophin signaling pathway & hsa04722 & 10 & 120 & 0.057179 & 0.133359 \\
\hline B cell receptor signaling pathway & hsa04662 & 7 & 73 & 0.058893 & 0.136606 \\
\hline Other glycan degradation & hsa00511 & 3 & 18 & 0.060693 & 0.138769 \\
\hline Platelet activation & hsa04611 & 10 & 122 & 0.06211 & 0.141916 \\
\hline Tight junction & hsa04530 & 11 & 139 & 0.062942 & 0.143409 \\
\hline Other types of O-glycan biosynthesis & hsa00514 & 4 & 31 & 0.063846 & 0.144608 \\
\hline Biosynthesis of amino acids & hsa01230 & 7 & 75 & 0.065542 & 0.147537 \\
\hline Propanoate metabolism & hsa00640 & 4 & 32 & 0.069465 & 0.151175 \\
\hline Carbohydrate digestion and absorption & hsa04973 & 5 & 46 & 0.069619 & 0.151258 \\
\hline RNA degradation & hsa03018 & 7 & 77 & 0.072625 & 0.157046 \\
\hline Hedgehog signaling pathway & hsa04340 & 5 & 47 & 0.074404 & 0.160515 \\
\hline Apoptosis - multiple species & hsa04215 & 4 & 33 & 0.075328 & 0.161634 \\
\hline Steroid biosynthesis & hsa00100 & 3 & 20 & 0.07587 & 0.161663 \\
\hline Cholinergic synapse & hsa04725 & 9 & 111 & 0.0779 & 0.165488 \\
\hline Valine leucine and isoleucine degradation & hsa00280 & 5 & 48 & 0.079364 & 0.166169 \\
\hline Circadian entrainment & hsa04713 & 8 & 95 & 0.079558 & 0.166169 \\
\hline Tyrosine metabolism & hsa00350 & 4 & 35 & 0.087772 & 0.179561 \\
\hline Phosphatidylinositol signaling system & hsa04070 & 8 & 98 & 0.09041 & 0.1845 \\
\hline Pancreatic cancer & hsa05212 & 6 & 66 & 0.092471 & 0.185911 \\
\hline Glycolysis / Gluconeogenesis & hsa00010 & 6 & 67 & 0.09726 & 0.193277 \\
\hline Mineral absorption & hsa04978 & 5 & 52 & 0.100902 & 0.19956 \\
\hline Endometrial cancer & hsa05213 & 5 & 52 & 0.100902 & 0.19956 \\
\hline Choline metabolism in cancer & hsa05231 & 8 & 101 & 0.102072 & 0.200801 \\
\hline Fc epsilon RI signaling pathway & hsa04664 & 6 & 68 & 0.102178 & 0.200801 \\
\hline Ubiquitin mediated proteolysis & hsa04120 & 10 & 137 & 0.107692 & 0.207883 \\
\hline Small cell lung cancer & hsa05222 & 7 & 86 & 0.109841 & 0.211319 \\
\hline Histidine metabolism & hsa00340 & 3 & 24 & 0.110548 & 0.211614 \\
\hline RIG-I-like receptor signaling pathway & hsa04622 & 6 & 70 & 0.112395 & 0.214746 \\
\hline $\begin{array}{c}\text { Chagas disease (American } \\
\text { trypanosomiasis) }\end{array}$ & hsa05142 & 8 & 104 & 0.114531 & 0.218011 \\
\hline
\end{tabular}


https://doi.org/10.33263/BRIAC111.79437972

\begin{tabular}{|c|c|c|c|c|c|}
\hline Thyroid hormone synthesis & hsa04918 & 6 & 71 & 0.11769 & 0.223052 \\
\hline T cell receptor signaling pathway & hsa04660 & 8 & 105 & 0.118859 & 0.22508 \\
\hline Gap junction & hsa04540 & 7 & 88 & 0.119267 & 0.225715 \\
\hline Fatty acid elongation & hsa00062 & 3 & 25 & 0.120021 & 0.225893 \\
\hline Pyruvate metabolism & hsa00620 & 4 & 40 & 0.122807 & 0.227762 \\
\hline Cell cycle & hsa04110 & 9 & 124 & 0.1254 & 0.232008 \\
\hline Chronic myeloid leukemia & hsa05220 & 6 & 73 & 0.128646 & 0.237773 \\
\hline Tuberculosis & hsa05152 & 12 & 179 & 0.128995 & 0.238371 \\
\hline NOD-like receptor signaling pathway & hsa04621 & 5 & 57 & 0.131451 & 0.240774 \\
\hline Fatty acid biosynthesis & hsa00061 & 2 & 13 & 0.136329 & 0.246461 \\
\hline Ascorbate and aldarate metabolism & hsa00053 & 3 & 27 & 0.139806 & 0.25082 \\
\hline Phototransduction & hsa04744 & 3 & 27 & 0.139806 & 0.25082 \\
\hline Platinum drug resistance & hsa01524 & 6 & 75 & 0.140073 & 0.251052 \\
\hline Fc gamma R-mediated phagocytosis & hsa04666 & 7 & 93 & 0.144571 & 0.258913 \\
\hline Glycerolipid metabolism & hsa00561 & 5 & 59 & 0.144711 & 0.259012 \\
\hline Serotonergic synapse & hsa04726 & 8 & 112 & 0.151497 & 0.26625 \\
\hline $\begin{array}{c}\text { Glycosphingolipid biosynthesis - globo } \\
\text { series }\end{array}$ & hsa00603 & 2 & 14 & 0.151522 & 0.26625 \\
\hline Long-term depression & hsa04730 & 5 & 60 & 0.151548 & 0.26625 \\
\hline Adrenergic signaling in cardiomyocytes & hsa04261 & 10 & 149 & 0.154941 & 0.271494 \\
\hline Carbon metabolism & hsa01200 & 8 & 113 & 0.156481 & 0.273723 \\
\hline Hippo signaling pathway -multiple species & hsa04392 & 3 & 29 & 0.160592 & 0.279422 \\
\hline Pancreatic secretion & hsa04972 & 7 & 96 & 0.160882 & 0.279661 \\
\hline $\begin{array}{l}\text { Non-alcoholic fatty liver disease } \\
\text { (NAFLD) }\end{array}$ & hsa04932 & 10 & 151 & 0.16369 & 0.28395 \\
\hline Colorectal cancer & hsa05210 & 5 & 62 & 0.165616 & 0.286802 \\
\hline Nicotinate and nicotinamide metabolism & hsa00760 & 3 & 30 & 0.171316 & 0.292913 \\
\hline Progesterone-mediated oocyte maturation & hsa04914 & 7 & 98 & 0.172196 & 0.294253 \\
\hline $\begin{array}{l}\text { Endocrine and other factor-regulated } \\
\text { calcium reabsorption }\end{array}$ & hsa04961 & 4 & 47 & 0.179927 & 0.306266 \\
\hline Sphingolipid metabolism & hsa00600 & 4 & 47 & 0.179927 & 0.306266 \\
\hline Mucin type O-Glycan biosynthesis & hsa00512 & 3 & 31 & 0.182236 & 0.306971 \\
\hline $\begin{array}{c}\text { Amino sugar and nucleotide sugar } \\
\text { metabolism }\end{array}$ & hsa00520 & 4 & 48 & 0.18872 & 0.316073 \\
\hline Type II diabetes mellitus & hsa04930 & 4 & 48 & 0.18872 & 0.316073 \\
\hline Vascular smooth muscle contraction & hsa04270 & 8 & 120 & 0.193437 & 0.322794 \\
\hline Long-term potentiation & hsa04720 & 5 & 66 & 0.19519 & 0.325542 \\
\hline Primary bile acid biosynthesis & hsa00120 & 2 & 17 & 0.198744 & 0.32872 \\
\hline Phenylalanine metabolism & hsa00360 & 2 & 17 & 0.198744 & 0.32872 \\
\hline Central carbon metabolism in cancer & hsa05230 & 5 & 67 & 0.202856 & 0.334977 \\
\hline Amyotrophic lateral sclerosis (ALS) & hsa05014 & 4 & 51 & 0.215869 & 0.349197 \\
\hline SNARE interactions in vesicular transport & hsa04130 & 3 & 34 & 0.216001 & 0.349197 \\
\hline Toll-like receptor signaling pathway & hsa04620 & 7 & 106 & 0.220603 & 0.354295 \\
\hline ErbB signaling pathway & hsa04012 & 6 & 88 & 0.224333 & 0.354295 \\
\hline GABAergic synapse & hsa04727 & 6 & 88 & 0.224333 & 0.354295 \\
\hline African trypanosomiasis & hsa05143 & 3 & 35 & 0.227536 & 0.354295 \\
\hline Salivary secretion & hsa04970 & 6 & 89 & 0.231416 & 0.354295 \\
\hline Cell adhesion molecules (CAMs) & hsa04514 & 9 & 146 & 0.232694 & 0.354295 \\
\hline Bile secretion & hsa04976 & 5 & 71 & 0.234456 & 0.354762 \\
\hline Pentose and glucuronate interconversions & hsa00040 & 3 & 36 & 0.239183 & 0.360503 \\
\hline PPAR signaling pathway & hsa03320 & 5 & 72 & 0.242564 & 0.36542 \\
\hline Rheumatoid arthritis & hsa05323 & 6 & 91 & 0.245786 & 0.369788 \\
\hline One carbon pool by folate & hsa00670 & 2 & 20 & 0.247388 & 0.370134 \\
\hline $\begin{array}{l}\text { Glycosaminoglycan biosynthesis - } \\
\text { chondroitin sulfate / dermatan sulfate }\end{array}$ & hsa00532 & 2 & 20 & 0.247388 & 0.370134 \\
\hline Pathogenic Escherichia coli infection & hsa05130 & 4 & 55 & 0.253542 & 0.377924 \\
\hline Fanconi anemia pathway & hsa03460 & 4 & 55 & 0.253542 & 0.377924 \\
\hline Adherens junction & hsa04520 & 5 & 74 & 0.258996 & 0.380865 \\
\hline NF-kappa B signaling pathway & hsa04064 & 6 & 93 & 0.260405 & 0.380865 \\
\hline Pertussis & hsa05133 & 5 & 75 & 0.267309 & 0.380927 \\
\hline Regulation of autophagy & hsa04140 & 3 & 40 & 0.286589 & 0.404693 \\
\hline Nicotine addiction & hsa05033 & 3 & 40 & 0.286589 & 0.404693 \\
\hline Bacterial invasion of epithelial cells & hsa05100 & 5 & 78 & 0.292575 & 0.406051 \\
\hline Antigen processing and presentation & hsa04612 & 5 & 78 & 0.292575 & 0.406051 \\
\hline Biosynthesis of unsaturated fatty acids & hsa01040 & 2 & 23 & 0.296367 & 0.406051 \\
\hline Bladder cancer & hsa05219 & 3 & 41 & 0.298573 & 0.406051 \\
\hline Oxytocin signaling pathway & hsa04921 & 9 & 158 & 0.301824 & 0.4095 \\
\hline VEGF signaling pathway & hsa04370 & 4 & 61 & 0.312149 & 0.420473 \\
\hline Retrograde endocannabinoid signaling & hsa04723 & 6 & 101 & 0.320797 & 0.428438 \\
\hline Arachidonic acid metabolism & hsa00590 & 4 & 62 & 0.32206 & 0.428438 \\
\hline Fatty acid degradation & hsa00071 & 3 & 44 & 0.33463 & 0.434229 \\
\hline Vasopressin-regulated water reabsorption & hsa04962 & 3 & 44 & 0.33463 & 0.434229 \\
\hline Taste transduction & hsa04742 & 5 & 83 & 0.335474 & 0.435078 \\
\hline Renin secretion & hsa04924 & 4 & 64 & 0.341941 & 0.443151 \\
\hline Maturity onset diabetes of the young & hsa04950 & 2 & 26 & 0.34484 & 0.445649 \\
\hline Ether lipid metabolism & hsa00565 & 3 & 45 & 0.346643 & 0.447475 \\
\hline
\end{tabular}

https://biointerfaceresearch.com/ 
https://doi.org/10.33263/BRIAC111.79437972

\begin{tabular}{|c|c|c|c|c|c|}
\hline Salmonella infection & hsa05132 & 5 & 86 & 0.361476 & 0.4536 \\
\hline Alzheimer's disease & hsa05010 & 9 & 168 & 0.362661 & 0.4549 \\
\hline Butanoate metabolism & hsa00650 & 2 & 28 & 0.376553 & 0.470076 \\
\hline Fatty acid metabolism & hsa01212 & 3 & 48 & 0.382511 & 0.472353 \\
\hline Dopaminergic synapse & hsa04728 & 7 & 130 & 0.385328 & 0.472353 \\
\hline $\begin{array}{l}\text { Synthesis and degradation of ketone } \\
\text { bodies }\end{array}$ & hsa00072 & 1 & 10 & 0.38641 & 0.472353 \\
\hline GnRH signaling pathway & hsa04912 & 5 & 91 & 0.404857 & 0.492475 \\
\hline Citrate cycle (TCA cycle) & hsa00020 & 2 & 30 & 0.40761 & 0.493684 \\
\hline Taurine and hypotaurine metabolism & hsa00430 & 1 & 11 & 0.413063 & 0.493684 \\
\hline Spliceosome & hsa03040 & 7 & 134 & 0.413895 & 0.494614 \\
\hline Glutathione metabolism & hsa00480 & 3 & 52 & 0.42957 & 0.510288 \\
\hline Glycerophospholipid metabolism & hsa00564 & 5 & 95 & 0.439305 & 0.512429 \\
\hline Base excision repair & hsa03410 & 2 & 33 & 0.452716 & 0.526293 \\
\hline Non-homologous end-joining & hsa03450 & 1 & 13 & 0.462947 & 0.530164 \\
\hline Prion diseases & hsa05020 & 2 & 35 & 0.481671 & 0.547988 \\
\hline Lysosome & hsa04142 & 6 & 123 & 0.490886 & 0.55257 \\
\hline Oocyte meiosis & hsa04114 & 6 & 123 & 0.490886 & 0.55257 \\
\hline $\begin{array}{c}\text { Glycosphingolipid biosynthesis - ganglio } \\
\text { series }\end{array}$ & hsa00604 & 1 & 15 & 0.508594 & 0.565358 \\
\hline Viral myocarditis & hsa05416 & 3 & 60 & 0.519209 & 0.575623 \\
\hline Hypertrophic cardiomyopathy (HCM) & hsa05410 & 4 & 83 & 0.525731 & 0.581799 \\
\hline Purine metabolism & hsa00230 & 8 & 176 & 0.549076 & 0.599938 \\
\hline Fat digestion and absorption & hsa04975 & 2 & 41 & 0.562575 & 0.612159 \\
\hline 2-Oxocarboxylic acid metabolism & hsa01210 & 1 & 18 & 0.569897 & 0.615889 \\
\hline Shigellosis & hsa05131 & 3 & 65 & 0.571183 & 0.616842 \\
\hline Porphyrin and chlorophyll metabolism & hsa00860 & 2 & 42 & 0.575147 & 0.620539 \\
\hline Dilated cardiomyopathy & hsa05414 & 4 & 90 & 0.587225 & 0.631028 \\
\hline Glycosaminoglycan degradation & hsa00531 & 1 & 19 & 0.588584 & 0.631028 \\
\hline $\begin{array}{l}\text { Epithelial cell signaling in Helicobacter } \\
\text { pylori infection }\end{array}$ & hsa05120 & 3 & 68 & 0.600639 & 0.642751 \\
\hline mRNA surveillance pathway & hsa03015 & 4 & 92 & 0.603916 & 0.646031 \\
\hline Drug metabolism - other enzymes & hsa00983 & 2 & 46 & 0.622767 & 0.661718 \\
\hline Arginine biosynthesis & hsa00220 & 1 & 21 & 0.623557 & 0.661718 \\
\hline $\begin{array}{c}\text { Metabolism of xenobiotics by cytochrome } \\
\text { P450 }\end{array}$ & hsa00980 & 3 & 73 & 0.646683 & 0.68192 \\
\hline Proximal tubule bicarbonate reclamation & hsa04964 & 1 & 23 & 0.655559 & 0.687708 \\
\hline Mismatch repair & hsa03430 & 1 & 23 & 0.655559 & 0.687708 \\
\hline N-Glycan biosynthesis & hsa00510 & 2 & 49 & 0.655695 & 0.687708 \\
\hline Vitamin digestion and absorption & hsa04977 & 1 & 24 & 0.670525 & 0.70006 \\
\hline alpha-Linolenic acid metabolism & hsa00592 & 1 & 25 & 0.684841 & 0.712655 \\
\hline Complement and coagulation cascades & hsa04610 & 3 & 79 & 0.696786 & 0.724031 \\
\hline Phagosome & hsa04145 & 6 & 155 & 0.704373 & 0.729833 \\
\hline Chemical carcinogenesis & hsa05204 & 3 & 82 & 0.719735 & 0.742898 \\
\hline Glyoxylate and dicarboxylate metabolism & hsa00630 & 1 & 28 & 0.724167 & 0.746632 \\
\hline Staphylococcus aureus infection & hsa05150 & 2 & 57 & 0.732262 & 0.754554 \\
\hline Linoleic acid metabolism & hsa00591 & 1 & 29 & 0.736154 & 0.757543 \\
\hline Steroid hormone biosynthesis & hsa00140 & 2 & 58 & 0.740738 & 0.76209 \\
\hline Galactose metabolism & hsa00052 & 1 & 31 & 0.758588 & 0.777663 \\
\hline Ribosome biogenesis in eukaryotes & hsa03008 & 3 & 89 & 0.767993 & 0.786689 \\
\hline RNA polymerase & hsa03020 & 1 & 32 & 0.769079 & 0.786923 \\
\hline Fructose and mannose metabolism & hsa00051 & 1 & 33 & 0.779115 & 0.79586 \\
\hline Morphine addiction & hsa05032 & 3 & 91 & 0.780469 & 0.797106 \\
\hline Cytosolic DNA-sensing pathway & hsa04623 & 2 & 64 & 0.786896 & 0.803182 \\
\hline Retinol metabolism & hsa00830 & 2 & 65 & 0.793845 & 0.809284 \\
\hline Inflammatory bowel disease (IBD) & hsa05321 & 2 & 66 & 0.800592 & 0.814985 \\
\hline Primary immunodeficiency & hsa05340 & 1 & 37 & 0.815085 & 0.827534 \\
\hline Drug metabolism - cytochrome P450 & hsa00982 & 2 & 69 & 0.81967 & 0.832006 \\
\hline Leishmaniasis & hsa05140 & 2 & 74 & 0.847849 & 0.857477 \\
\hline Gastric acid secretion & hsa04971 & 2 & 74 & 0.847849 & 0.857477 \\
\hline $\begin{array}{l}\text { Arrhythmogenic right ventricular } \\
\text { cardiomyopathy (ARVC) }\end{array}$ & hsa05412 & 2 & 74 & 0.847849 & 0.857477 \\
\hline Pyrimidine metabolism & hsa00240 & 3 & 105 & 0.853017 & 0.862133 \\
\hline Basal transcription factors & hsa03022 & 1 & 45 & 0.870412 & 0.878553 \\
\hline Parkinson's disease & hsa05012 & 4 & 142 & 0.882593 & 0.889869 \\
\hline Malaria & hsa05144 & 1 & 49 & 0.89152 & 0.898179 \\
\hline $\begin{array}{l}\text { Intestinal immune network for IgA } \\
\text { production }\end{array}$ & hsa04672 & 1 & 50 & 0.896237 & 0.902732 \\
\hline Autoimmune thyroid disease & hsa05320 & 1 & 54 & 0.91314 & 0.918749 \\
\hline Starch and sucrose metabolism & hsa00500 & 1 & 57 & 0.923986 & 0.929152 \\
\hline Ribosome & hsa03010 & 3 & 138 & 0.947254 & 0.950674 \\
\hline Olfactory transduction & hsa04740 & 2 & 420 & 1 & 1 \\
\hline
\end{tabular}

